\title{
Model-independent superhedging under portfolio constraints
}

\author{
Arash Fahim · Yu-Jui Huang
}

June 16, 2015

\begin{abstract}
In a discrete-time market, we study model-independent superhedging, while the semi-static superhedging portfolio consists of three parts: static positions in liquidly traded vanilla calls, static positions in other tradable, yet possibly less liquid, exotic options, and a dynamic trading strategy in risky assets under certain constraints. By considering the limit order book of each tradable exotic option and employing the Monge-Kantorovich theory of optimal transport, we establish a general superhedging duality, which admits a natural connection to convex risk measures. With the aid of this duality, we derive a model-independent version of the fundamental theorem of asset pricing. The notion "finite optimal arbitrage profit", weaker than no-arbitrage, is also introduced. It is worth noting that our method covers a large class of Delta constraints as well as Gamma constraint.
\end{abstract}

Keywords model-independent pricing - robust superhedging - limit order book · fundamental theorem of asset pricing $\cdot$ portfolio constraints $\cdot$ Monge-Kantorovich optimal transport

Mathematics Subject Classification (2010) 91G20 • 91G80

JEL Classification C61 - G13

A. Fahim

Department of Mathematics, Florida State University

E-mail: fahim@math.fsu.edu

Y.-J. Huang

School of Mathematical Sciences, Dublin City University

E-mail: yujui.huang@dcu.ie

We thank Pierre Henry-Labordère, Constantinos Kardaras, and Jan Obłój for their thoughtful suggestions. We are also thankful to the anonymous referees for their elaborate comments which contribute to the quality of this work.

A. Fahim is partially supported by Florida State University CRC FYAP (315-81000-2424) and the NSF (DMS-1209519).

Y.-J. Huang is partially supported by SFI (07/MI/008 and 08/SRC/FMC1389) and the ERC (278295). 


\section{Introduction}

To avoid model mis-specification, one may choose to consider only "must-be-true" implications from the market. The standard approach, suggested by Dupire [16], leverages on market prices of liquidly traded vanilla call options: one does not manage to specify a proper physical measure, but considers all measures that are consistent with market prices of vanilla calls as plausible pricing measures. These measures then provide model-independent bounds for prices of illiquid exotic options, and motivate the practically useful semi-static hedging, which involves static holdings in vanilla calls and dynamic trading in risky assets. Pioneered by Hobson [25], this thread of research has drawn substantial attention; see e.g. [8], [6], [27], [26], [30], [11], [12], [5], and [14]. In particular, Beiglböck, Henry-Labordère \& Penkner establish in [5] a general duality of model-independent superhedging, under a discrete-time setting where market prices of vanilla calls with maturities at or before the terminal time $T>0$ are all considered.

In reality, what we can rely on goes beyond vanilla calls. In the markets of commodities, for instance, Asian options and calendar spread options are largely traded, with their market or broker quotes easily accessible. In the New York Stock Exchange and the Chicago Board Options Exchange, standardized digital and barrier options have been introduced, mostly for equity indexes and Exchange Traded Funds. What we can take advantage of, as a result, includes market prices of not only vanilla calls, but also certain tradable exotic options.

In this paper, we take up the model-independent framework in [5], and intend to establish a general superhedging duality, under the consideration of additional tradable options besides vanilla calls, as well as portfolio constraints on trading strategies in risky assets. More specifically, our semi-static superhedging portfolio consists of three parts: 1 . static positions in liquidly traded vanilla calls, as in the literature of robust hedging; 2 . static positions in additional tradable, yet possibly less liquid, exotic options; 3 . a dynamic trading strategy in risky assets under certain constraints.

While tradable, the additional exotic options may be very different from vanilla calls, in terms of liquidity. Their limit order books are usually very shallow and admit large bid-ask spreads, compared to those of the underlying assets and the associated vanilla calls. It follows that we need to take into account the whole limit order book, instead of one single market quote, of each of the additional options, in order to make trading possible. We formulate the limit order books in Section 2.1 and consider the corresponding non-constant unit price functions. On the other hand, portfolio constraints on trading strategies in risky assets have been widely studied under the model-specific case; see [13] and [28] for deterministic convex constraints, and [19], [9], [32], and [33], among others, for random and other more general constraints. Our goal is to place portfolio constraints under current model-independent context, and investigate its implication to semi-static superhedging.

We particularly consider a general class of constraints which enjoys adapted convexity and continuous approximation property (Definition 2.7). This already covers a large collection of Delta constraints, including adapted convex constraints; see Remark 2.9. For the simpler case where no additional tradable option exists, we derive a superhedging duality in Proposition 3.10 by using the theory of optimal transport. 
This in particular generalizes the duality in [5] to the multi-dimensional case with portfolio constraints; see Remarks 3.12 and 3.13. Then, on strength of the convexity of the non-constant unit price functions, we are able to extend the above duality to the general case where additional tradable options exist; see Theorem 3.14 Note that Acciaio, Beiglböck, Penkner \& Schachermayer [1] also applies to model-independent superhedging in the presence of tradable exotic options, while assuming implicitly that each option can be traded liquidly. Theorem 3.14 can therefore be seen as a generalization of [1] that deals with different levels of liquidity; see Remark 3.16

The second part of the paper investigates the relation between the superhedging duality and the fundamental theorem of asset pricing (FTAP). It is well known in the classical model-specific case that the FTAP yields the superhedging duality. This relation has been carried over to the model-independent case by [1], where an appropriate notion of model-independent arbitrage was introduced. In the same spirit as in [1], we define model-independent arbitrage in Definition 4.1, under current setting with additional tradable options and portfolio constraints. With the aid of the superhedging duality in Theorem 3.14 we are able to derive a model-independent FTAP; see Theorem 4.8 While the theorem itself does not distinguish between arbitrage due to risky assets and arbitrage due to additional tradable options, Lemmas 4.4 and 4.6 can be used to differentiate one from the other. It is also worth noting that we derive the FTAP as a consequence of the superhedging duality. This argument was first observed in [15], as opposed to the standard argument of deriving the superhedging duality as a consequence of the FTAP, used in both the model-specific case and [1].

With the FTAP (Theorem 4.8) at hand, we observe from Theorem 3.14 and Proposition 3.17 that the problems of superhedging and risk-measuring can be well-defined even when there is model-independent arbitrage to some extent. We relate this to optimal arbitrage under the formulation of [10], and show that superhedging and riskmeasuring are well-defined as long as "the optimal arbitrage profit is finite", a notion weaker than no-arbitrage; see Proposition 4.15 We also compare Theorem 4.8 with [21, Theorem 9.9], the classical model-specific FTAP under portfolio constraints, and observe that a closedness condition in [21] is no longer needed under current setting. An example given in Section 4.1 indicates that availability of vanilla calls obviates the need of the closedness condition.

Finally, we extend our scope to Gamma constraint. While Gamma constraint does not satisfy adapted convexity in Definition 2.7(ii), it admits additional boundedness property. Taking advantage of this, we are able to modify previous results to obtain the corresponding superhedging duality and FTAP in Propositions 6.3 and 6.6

This paper is organized as follows. In Section 2, we prescribe the set-up of our studies. In Section 3, we establish the superhedging duality, and investigate its connection to other dualities in the literature and convex risk measures. In Section 4 , we define model-independent arbitrage under portfolio constraints with additional tradable options, and derive the associated FTAP. The notion "finite optimal arbitrage profit", weaker than no-arbitrage, is also introduced. Section 5 presents concrete examples of portfolio constraints and the effect of additional tradable options. Section 6 deals with constraints which do not enjoy adapted convexity, but admit some boundedness property. Appendix $\AA$ contains a counter-example which emphasizes the necessity of the continuous approximation property required in Definition 2.7 


\section{The set-up}

We consider a discrete-time market, with a finite horizon $T \in \mathbb{N}$. There are $d$ risky assets $S=\left\{S_{t}\right\}_{t=0}^{T}=\left\{\left(S_{t}^{1}, \ldots, S_{t}^{d}\right)\right\}_{t=0}^{T}$, whose initial price $S_{0}=x_{0} \in \mathbb{R}_{+}^{d}$ is given. There is also a risk-free asset $B=\left\{B_{t}\right\}_{t=0}^{T}$ which is normalized to $B_{t} \equiv 1$. Specifically, we take $S$ as the canonical process $S_{t}\left(x_{1}, x_{2}, \ldots, x_{T}\right)=x_{t}$ on the path-space $\Omega:=\left(\mathbb{R}_{+}^{d}\right)^{T}$, and denote by $\mathbb{F}=\left\{\mathscr{F}_{t}\right\}_{t=0}^{T}$ the natural filtration generated by $S$.

\subsection{Vanilla calls and other tradable options}

At time 0 , we assume that the vanilla call option with payoff $\left(S_{t}^{n}-K\right)^{+}$can be liquidly traded, at some price $C_{n}(t, K)$ given in the market, for all $n=1, \ldots, d, t=1, \ldots, T$, and $K \geq 0$. The collection of pricing measures consistent with market prices of vanilla calls is therefore

$\Pi:=\left\{\mathbb{Q} \in \mathscr{P}(\Omega): \mathbb{E}^{\mathbb{Q}}\left[\left(S_{t}^{n}-K\right)^{+}\right]=C_{n}(t, K), \forall n=1, \ldots, d, t=1, \ldots, T\right.$, and $\left.K \geq 0\right\}$,

where $\mathscr{P}(\Omega)$ denotes the collection of all probability measures defined on $\Omega$.

In view of [24, Proposition 2.1], for each $n=1, \ldots, d$ and $t=1, \ldots, T$, as long as $K \mapsto C_{n}(t, K)$ is nonnegative, convex and satisfies $\lim _{K \downarrow 0} \partial_{K} C_{n}(t, K) \geq-1$, and $\lim _{K \rightarrow \infty} C_{n}(t, K)=0$, the relation $\mathbb{E}^{\mathbb{Q}}\left[\left(S_{t}^{n}-K\right)^{+}\right]=C_{n}(t, K) \forall K \geq 0$ already prescribes the distribution of $S_{t}^{n}$ on $\mathbb{R}_{+}$, which will be denoted by $\mu_{t}^{n}$. Thus, by setting $\mathbb{Q}_{t}^{n}$ as the law of $S_{t}^{n}$ under $\mathbb{Q}$, we have

$$
\Pi=\left\{\mathbb{Q} \in \mathscr{P}(\Omega): \mathbb{Q}_{t}^{n}=\mu_{t}^{n}, \forall n=1, \ldots, d \text { and } t=1, \ldots, T\right\} .
$$

Remark 2.1 Given $\mathbb{Q} \in \Pi$, note that $\mathbb{E}^{\mathbb{Q}}\left[S_{t}^{n}\right]<\infty$ for all $n=1, \ldots, d$ and $t=1, \ldots, T$ (which can be seen by taking $K=0$ in (2.1)).

Remark 2.2 In view of (2.2), $\Pi$ is nonempty, convex, and weakly compact. This is a direct consequence of [29. Proposition 1.2], once we view $\Omega=\left(\mathbb{R}_{+}^{d}\right)^{T}$ as the product of $(d \times T)$ copies of $\mathbb{R}_{+}$.

Remark 2.3 We do not assume that $t \mapsto C_{n}(t, K)$ is increasing for each fixed $n$ and $K$. This condition, normally required in the literature (see e.g. [5] p. 481]), implies that the set of martingale measures

$$
\mathscr{M}:=\left\{\mathbb{Q} \in \Pi: S=\left\{S_{t}\right\}_{t=0}^{T} \text { is a martingale under } \mathbb{Q}\right\}
$$

is non-empty, which underlies the superhedging duality in [5]. In contrast, the superhedging duality in Proposition 3.10 below hinges on a different collection $\mathscr{Q}_{\mathscr{S}}$ which contains $\mathscr{M}$ (see Definition 3.4). Since it is possible that our duality holds while $\mathscr{M}=\emptyset$, imposing “ $t \mapsto C_{n}(t, K)$ is increasing” is not necessary.

Besides vanilla calls, there are other options tradable, while less liquid, at time 0 . Let $I$ be a (possibly uncountable) index set. For each $i \in I$, suppose that $\psi_{i}: \Omega \mapsto \mathbb{R}$ is the payoff function of an option tradable at time 0 . Let $\eta \in \mathbb{R}$ be the number of units of $\psi_{i}$ being traded at time 0 , with $\eta \geq 0$ denoting a purchase order and $\eta<0$ a 
selling order. Let $c_{i}(\eta) \in \overline{\mathbb{R}}:=\mathbb{R} \cup\{-\infty,+\infty\}$ denote the total cost of trading $\eta$ units of $\psi_{i}$. Throughout this paper, we impose the following condition:

$$
\text { for all } i \in I \text {, the map } \eta \mapsto c_{i}(\eta) \text { is convex with } c_{i}(0)=0 \text {. }
$$

We can then define the unit price $p_{i}(\eta)$ for trading $\eta$ units of $\psi_{i}$ by

$$
p_{i}(\eta):=\frac{c_{i}(\eta)}{\eta} \quad \text { for } \eta \in \mathbb{R} \backslash\{0\}, \quad \text { and } \quad p_{i}(0):=c_{i}^{\prime}(0+) \text {. }
$$

Remark 2.4 Condition $(C)$ is motivated by the typical structure of a limit order book of a nonnegative option, as demonstrated in Figure 2.1] That is, the option $\psi_{i}$ can be purchased only at prices $0 \leq a_{1} \leq a_{2} \leq \cdots \leq a_{\ell}$ with number of units $q_{1}, q_{2}, \ldots, q_{\ell}>0$ respectively, and sold only at prices $b_{1} \geq b_{2} \geq \cdots \geq b_{k} \geq 0$ with number of units $r_{1}, r_{2}, \ldots, r_{k}>0$ respectively, where $b_{1} \leq a_{1}$ reflects the bid-ask spread and $\ell$ and $k$ belong to $\mathbb{N} \cup\{+\infty\}$. The possibility of $\ell, k=\infty$ allows for infinitely many buy/sell prices in the order book.

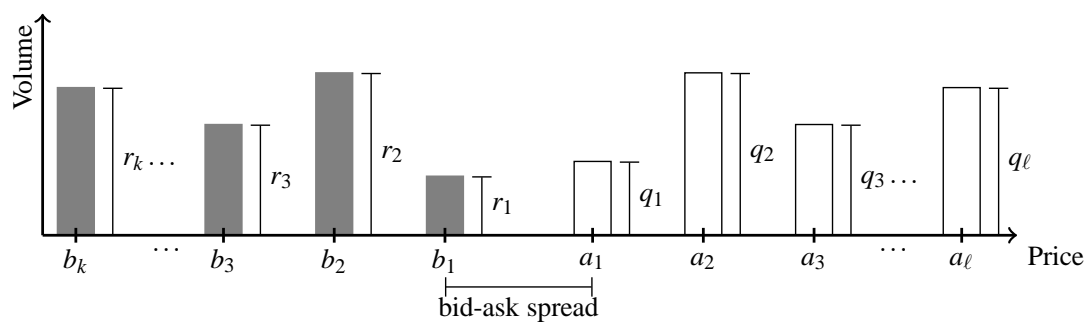

Fig. 2.1 A limit order book of $\psi_{i}$

We keep track of $Q_{m}:=\sum_{j=1}^{m} q_{j}$, the total number of units that can be bought at or below the price $a_{m}$, for $m=1, \ldots, \ell$. Similarly, $R_{m}:=\sum_{j=1}^{m} r_{j}$ is the total number of units that can be sold at or above the price $b_{m}$, for all $m=1, \ldots, k$. The total cost $c_{i}(\eta)$ of trading $\eta$ units of $\psi_{i}$ is then given by

$c_{i}(\eta)= \begin{cases}\sum_{m=1}^{u-1} a_{m} q_{m}+a_{u}\left(\eta-Q_{u-1}\right) \geq 0 & \text { if } \eta \in\left(Q_{u-1}, Q_{u}\right], u=1, \ldots, \ell+1 . \\ 0 & \text { if } \eta=0, \\ -\sum_{m=1}^{u-1} b_{m} r_{m}+b_{u}\left(\eta+R_{u-1}\right) \leq 0 & \text { if } \eta \in\left[-R_{u},-R_{u-1}\right), u=0, \ldots, k+1 .\end{cases}$

where we set $Q_{0}=R_{0}=0, Q_{\ell+1}=R_{k+1}=\infty, a_{\ell+1}=\infty, b_{k+1}=0$, and use the convention that $0 \cdot \infty=0$ and $\sum_{m=1}^{0}=0$. As shown in Figure 2.2 $\eta \mapsto c_{i}(\eta)$ satisfies (C). In particular, $c_{i}$ is linear on $\mathbb{R}$ if and only if $b_{1}=a_{1}$ and $q_{1}=r_{1}=\infty$; this means that $\psi_{i}$ can be traded liquidly at the price $a_{1}=b_{1}$, which is the slope of $c_{i}$.

Remark 2.5 Condition $(C)$ captures two important features of the prices of $\psi_{i}:$ (1) the bid-ask spread, formulated as $\left[c_{i}^{\prime}(0-), c_{i}^{\prime}(0+)\right] ;(2)$ the non-linearity, i.e. the unit price $\eta \mapsto p_{i}(\eta)$ is non-constant. This setting in particular allows for zero spread when $c_{i}^{\prime}(0-)=c_{i}^{\prime}(0+)$, while at the same time the limit order book may induce nonlinear pricing. This happens to a highly liquid asset for which the bid-ask spread is 


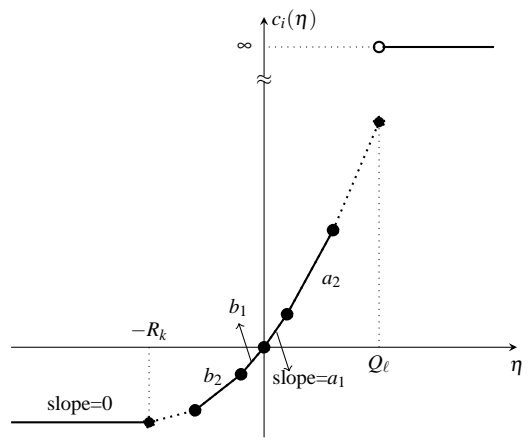

Fig. 2.2 Graph of $\eta \mapsto c_{i}(\eta): a_{1}, a_{2}, \ldots$ and $b_{1}, b_{2}, \ldots$ placed on each segment indicates the slope of each segment and matches the prices in the limit order book for volumes $q_{1}, q_{2}, \ldots$ and $r_{1}, r_{2}, \ldots$, respectively.

negligible, but transaction cost becomes significant for large trading volumes. Also, $c_{i}$ is linear if and only if $\psi_{i}$ can be traded liquidly, with whatever units, at one single price $p_{i}$ (which is the slope of $c_{i}$ ).

Note that [3] has recently considered bid-ask spreads, but not non-linear pricing, of hedging options under model uncertainty. In a model-independent setting, while a non-linear pricing operator for hedging options has been used in [15], the nonlinearity does not reflect the non-constant unit price of an option in its limit order book (see [15] (2.3)]); instead, it captures a market where the price of a portfolio of options may be lower than the sum of the respective prices of the options (see the second line in the proof of [15] Lemma 2.4]).

\subsection{Constrained trading strategies}

Definition 2.6 (Trading strategies) We say $\Delta=\left\{\Delta_{t}\right\}_{t=0}^{T-1}$ is a trading strategy if $\Delta_{0} \in \mathbb{R}^{d}$ is a constant and $\Delta_{t}:\left(\mathbb{R}_{+}^{d}\right)^{t} \mapsto \mathbb{R}^{d}$ is Borel measurable for all $t=1, \ldots, T-1$. Moreover, the stochastic integral of $\Delta$ with respect to $x=\left(x_{1}, \ldots, x_{T}\right) \in\left(\mathbb{R}_{+}^{d}\right)^{T}$ will be expressed as

$$
(\Delta \cdot x)_{t}:=\sum_{i=0}^{t-1} \Delta_{i}\left(x_{1}, \ldots, x_{i}\right) \cdot\left(x_{i+1}-x_{i}\right), \text { for } t=1, \ldots, T
$$

where in the right hand side above, $\Delta_{i}=\left(\Delta_{i}^{1}, \ldots, \Delta_{i}^{d}\right), x_{i}=\left(x_{i}^{1}, \ldots, x_{i}^{d}\right)$, and " ." denotes the inner product in $\mathbb{R}^{d}$. We will denote by $\mathscr{H}$ the collection of all trading strategies. Also, for any collection $\mathscr{J} \subseteq \mathscr{H}$, we introduce the sub-collections

$$
\begin{aligned}
\mathscr{J}^{\infty} & :=\left\{\Delta \in \mathscr{J}: \Delta_{t}:\left(\mathbb{R}_{+}^{d}\right)^{t} \mapsto \mathbb{R}^{d} \text { is bounded, } \forall t=1, \ldots, T-1\right\}, \\
\mathscr{J}_{c}^{\infty}: & =\left\{\Delta \in \mathscr{J}^{\infty}: \Delta_{t}:\left(\mathbb{R}_{+}^{d}\right)^{t} \mapsto \mathbb{R}^{d} \text { is continuous, } \forall t=1, \ldots, T-1\right\} .
\end{aligned}
$$

In this paper, we require the trading strategies to lie in a sub-collection $\mathscr{S}$ of $\mathscr{H}$, prescribed as below. 
Definition 2.7 (Adaptively convex portfolio constraint) $\mathscr{S}$ is a set of trading strategies such that

(i) $0 \in \mathscr{S}$.

(ii) For any $\Delta, \Delta^{\prime} \in \mathscr{S}$ and adapted process $h$ with $h_{t} \in[0,1]$ for all $t=0, \ldots, T-1$,

$$
\left\{h_{t} \Delta_{t}+\left(1-h_{t}\right) \Delta_{t}^{\prime}\right\}_{t=0}^{T-1} \in \mathscr{S} \text {. }
$$

(iii) For any $\Delta \in \mathscr{S}^{\infty}, \mathbb{Q} \in \Pi$, and $\varepsilon>0$, there exist a closed set $D_{\varepsilon} \subseteq\left(\mathbb{R}_{+}^{d}\right)^{T}$ and $\Delta^{\varepsilon} \in \mathscr{S}_{c}^{\infty}$ such that

$$
\mathbb{Q}\left(D_{\varepsilon}\right)>1-\varepsilon \text { and } \Delta_{t}=\Delta_{t}^{\varepsilon} \text { on } D_{\varepsilon} \text { for } t=0, \ldots, T-1 .
$$

Remark 2.8 In Definition 2.7 ( $i$ ) and (ii) are motivated by [21. Section 9.1], while (iii) is a technical assumption which allows us to perform continuous approximation in Lemma 3.3. This approximation in particular enables us to establish the superhedging duality in Proposition 3.10. In fact, if we only have conditions (i) and (ii), the duality in Proposition 3.10 may fail in general, as demonstrated in Appendix $A$

An explained below, Definition 2.7(iii) covers a large class of convex constraints.

Remark 2.9 (Adapted convex constraints) Let $\left\{K_{t}\right\}_{t=0}^{T-1}$ be an adapted set-valued process such that for each $t, K_{t}$ maps $\left(x_{1}, \ldots, x_{t}\right) \in\left(\mathbb{R}_{+}^{d}\right)^{t}$ to a closed convex set $K_{t}\left(x_{1}, \ldots, x_{t}\right) \subseteq \mathbb{R}^{d}$ which contains 0 . Consider the collection of trading strategies

$\mathscr{S}:=\left\{\Delta \in \mathscr{H}:\right.$ for each $\left.t \geq 0, \Delta_{t}\left(x_{1}, \ldots, x_{t}\right) \in K_{t}\left(x_{1}, \ldots, x_{t}\right), \forall\left(x_{1}, \ldots, x_{t}\right) \in\left(\mathbb{R}_{+}^{d}\right)^{t}\right\}$,

which satisfies Definition 2.7 ( $i$ ) and (ii) trivially. To obtain Definition 2.7 (iii), we assume additionally that for each $t \geq 1$, the set-valued map $K_{t}:\left(\mathbb{R}_{+}^{d}\right)^{t} \mapsto 2^{\mathbb{R}^{d}}$ is lower semicontinuous, in the sense that

$$
\text { for any } V \text { open in } \mathbb{R}^{d} \text {, the set }\left\{x \in\left(\mathbb{R}_{+}^{d}\right)^{t}: K_{t}(x) \cap V \neq \emptyset\right\} \text { is open in }\left(\mathbb{R}_{+}^{d}\right)^{t} \text {. }
$$

This is equivalent to the following condition:

$$
\begin{aligned}
& \forall y_{0} \in K_{t}\left(x_{0}\right) \text { and }\left\{x_{n}\right\} \subset\left(\mathbb{R}_{+}^{d}\right)^{t} \text { such that } x_{n} \rightarrow x_{0}, \\
& \exists y_{n} \in K_{t}\left(x_{n}\right) \text { such that } y_{n} \rightarrow y_{0} ;
\end{aligned}
$$

see e.g. [2. Definition 1.4.2] and the remark below it, and [22] Section 2.5].

To check (iii), let us fix $\Delta \in \mathscr{S}^{\infty}, \mathbb{Q} \in \Pi$, and $\varepsilon>0$. For each $t=1, \ldots, T-1$, by Lusin's theorem, there exists a closed set $D_{\varepsilon, t} \subset\left(\mathbb{R}_{+}^{d}\right)^{t}$ such that $\left.\Delta_{t}\right|_{D_{\varepsilon, t}}: D_{\varepsilon, t} \mapsto K_{t}$ is continuous and $\mathbb{Q}\left(D_{\varepsilon, t} \times\left(\mathbb{R}_{+}^{d}\right)^{T-t}\right)>1-\frac{\varepsilon}{T-1}$. Under $(2.5)$, we can apply the theory of continuous selection (see e.g. [31. Theorem 3.2" ]) to find a bounded continuous function $\Delta_{t}^{\varepsilon}:\left(\mathbb{R}_{+}^{d}\right)^{t} \mapsto K_{t}$ such that $\Delta_{t}^{\varepsilon}=\Delta_{t}$ on $D_{\varepsilon, t}$. Now, set $\Delta_{0}^{\varepsilon}=\Delta_{0}$, and define $D_{\varepsilon}:=\bigcap_{t} D_{\varepsilon, t} \times\left(\mathbb{R}_{+}^{d}\right)^{T-t}$, which is by definition closed in $\mathbb{R}_{+}^{T}$. We see that $\Delta^{\varepsilon} \in \mathscr{S}_{c}^{\infty}$, $\mathbb{Q}\left(D_{\varepsilon}\right)>1-\varepsilon$, and $\Delta_{t}=\Delta_{t}^{\varepsilon}$ on $D_{\varepsilon}$ for all $t=0, \ldots, T-1$. This already verifies Definition 2.7(iii).

Note that for the special case where $\left\{K_{t}\right\}_{t=1}^{T}$ is deterministic, $K_{t}$ is a fixed subset of $\mathbb{R}^{d}$ for each $t$ and thus (2.6) is trivially satisfied. See Examples 5.2 and 5.3 below for a concrete illustration of deterministic and adapted convex constraints. 


\section{The superhedging duality}

For a path-dependent exotic option with payoff function $\Phi:\left(\mathbb{R}_{+}^{d}\right)^{T} \mapsto \mathbb{R}$, we intend to construct a semi-static superhedging portfolio, which consists of three parts: static positions in vanilla calls, static positions in $\left\{\psi_{i}\right\}_{i \in I}$, and a dynamic trading strategy $\Delta \in \mathscr{S}$. More precisely, consider

$$
\begin{aligned}
\mathscr{C} & :=\left\{\varphi: \mathbb{R} \mapsto \mathbb{R}: \varphi(x)=a+\sum_{i=1}^{n} b_{i}\left(x-K_{i}\right)^{+} \text {with } a \in \mathbb{R}, n \in \mathbb{N}, b_{i} \in \mathbb{R}, K_{i}>0\right\}, \\
\mathscr{R}^{I} & :=\left\{\eta=\left(\eta_{i}\right)_{i \in I} \in \mathbb{R}: \eta_{i} \neq 0 \text { for finitely many } i \text { 's }\right\} .
\end{aligned}
$$

We intend to find $u=\left\{u_{t}^{n} \in \mathscr{C}: n=1, \ldots, d ; t=1, \ldots, T\right\}, \eta \in \mathscr{R}^{I}$, and $\Delta \in \mathscr{S}$ such that

$$
\Psi_{u, \eta, \Delta}(x):=\sum_{t=1}^{T} \sum_{n=1}^{d} u_{t}^{n}\left(x_{t}^{n}\right)+\sum_{i \in I}\left(\eta_{i} \psi_{i}-c_{i}\left(\eta_{i}\right)\right)+(\Delta \cdot x)_{T} \geq \Phi(x) \forall x \in\left(\mathbb{R}_{+}^{d}\right)^{T}
$$

where we assume that $0 \cdot \infty=0$. In the definition of $\mathscr{C}$, we specifically require $K_{i}$ to be strictly positive. This is because $K_{i}=0$ corresponds to trading the risky assets, which is already incorporated into $\Delta \in \mathscr{S}$ and should not be treated as part of the static positions. By setting $\mathscr{U}$ as the collection of all $u=\left\{u_{t}^{n} \in \mathscr{C}: n=1, \ldots, d, t=1, \ldots, T\right\}$, we define the superhedging price of $\Phi$ by

$$
\begin{aligned}
D(\Phi):=\inf \left\{\sum_{t=1}^{T} \sum_{n=1}^{d} \int_{\mathbb{R}_{+}} u_{t}^{n} d \mu_{t}^{n}: u \in \mathscr{U},\right. & \eta \in \mathscr{R}^{I} \text { and } \Delta \in \mathscr{S} \\
& \text { satisfy } \left.\Psi_{u, \eta, \Delta} \geq \Phi, \forall x \in\left(\mathbb{R}_{+}^{d}\right)^{T}\right\} .
\end{aligned}
$$

By introducing $\mathscr{U}_{0}:=\left\{u \in \mathscr{U}: \sum_{t=1}^{T} \sum_{n=1}^{d} \int_{\mathbb{R}_{+}} u_{t}^{n} d \mu_{t}^{n}=0\right\}$, we may express (3.2) as $D(\Phi)=\inf \left\{a \in \mathbb{R}: u \in \mathscr{U}_{0}, \eta \in \mathscr{R}^{I}\right.$ and $\Delta \in \mathscr{S}$ satisfy $\left.a+\Psi_{u, \eta, \Delta} \geq \Phi, \forall x \in\left(\mathbb{R}_{+}^{d}\right)^{T}\right\}$

Our goal in this section is to derive a superhedging duality associated with $D(\Phi)$.

\subsection{Upper variation process}

In order to deal with the portfolio constraint $\mathscr{S}$, we introduce an auxiliary process for each $\mathbb{Q} \in \Pi$, as suggested in [21, Section 9.2].

Definition 3.1 Given $\mathbb{Q} \in \Pi$, the upper variation process $A^{\mathbb{Q}}$ for $\mathscr{S}$ is defined by $A_{0}^{\mathbb{Q}}:=0$, and $A_{t+1}^{\mathbb{Q}}-A_{t}^{\mathbb{Q}}:=\underset{\Delta \in \mathscr{S}}{\operatorname{esssup}} \mathbb{Q}\left\{\Delta_{t} \cdot\left(\mathbb{E}^{\mathbb{Q}}\left[S_{t+1} \mid \mathscr{F}_{t}\right]-S_{t}\right)\right\}, t=0, \ldots, T-1$. 
First, Note that the conditional expectation in the definition of $A^{\mathbb{Q}}$ is well-defined, thanks to Remark 2.1. Next, since Definition 2.7(i)-(ii) implies $1_{\{|\Delta| \leq n\}} \Delta \in \mathscr{S}$ whenever $\Delta \in \mathscr{S}$, we may replace $\mathscr{S}$ by $\mathscr{S}^{\infty}$ in the above definition. It follows that

$$
A_{t+1}^{\mathbb{Q}}-A_{t}^{\mathbb{Q}}=\underset{\Delta \in \mathscr{S}^{\infty}}{\operatorname{ess} \sup ^{\mathbb{Q}}} \mathbb{E}^{\mathbb{Q}}\left[\Delta_{t} \cdot\left(S_{t+1}-S_{t}\right) \mid \mathscr{F}_{t}\right], t=0, \ldots, T-1 .
$$

Therefore,

$$
A_{t}^{\mathbb{Q}}=\sum_{i=1}^{t} \operatorname{ess~sup}_{\Delta \in \mathscr{S}^{\infty}} \mathbb{Q}^{\mathbb{Q}}\left[\Delta_{i-1} \cdot\left(S_{i}-S_{i-1}\right) \mid \mathscr{F}_{i-1}\right], \quad t=1, \ldots, T .
$$

Lemma 3.2 For any $\mathbb{Q} \in \Pi$ and $t=1, \ldots, T$, we have

$$
\mathbb{E}^{\mathbb{Q}}\left[\underset{\Delta \in \mathscr{S}^{\infty}}{\operatorname{ess} \sup ^{\mathbb{Q}}} \mathbb{E}^{\mathbb{Q}}\left[\Delta_{t} \cdot\left(S_{t+1}-S_{t}\right) \mid \mathscr{F}_{t}\right]\right]=\sup _{\Delta \in \mathscr{S}^{\infty}} \mathbb{E}^{\mathbb{Q}}\left[\Delta_{t} \cdot\left(S_{t+1}-S_{t}\right)\right] .
$$

This in particular implies that

$$
\mathbb{E}^{\mathbb{Q}}\left[A_{t}^{\mathbb{Q}}\right]=\sup _{\Delta \in \mathscr{S}^{\infty}} \mathbb{E}^{\mathbb{Q}}\left[(\Delta \cdot S)_{t}\right]
$$

Proof. First, note that $\left\{\mathbb{E}^{\mathbb{Q}}\left[\Delta_{t} \cdot\left(S_{t+1}-S_{t}\right) \mid \mathscr{F}_{t}\right]: \Delta \in \mathscr{S}^{\infty}\right\}$ is directed upwards. Indeed, given $\Delta, \Delta^{\prime} \in \mathscr{S}^{\infty}$, define $\tilde{\Delta}_{s}:=\Delta_{s} 1_{\{s \neq t\}}+\left(\Delta_{s} 1_{A}+\Delta_{s}^{\prime} 1_{A^{c}}\right) 1_{\{s=t\}}$, where

$$
A:=\left\{\mathbb{E}^{\mathbb{Q}}\left[\Delta_{t} \cdot\left(S_{t+1}-S_{t}\right) \mid \mathscr{F}_{t}\right] \geq \mathbb{E}^{\mathbb{Q}}\left[\Delta_{t}^{\prime} \cdot\left(S_{t+1}-S_{t}\right) \mid \mathscr{F}_{t}\right]\right\} \in \mathscr{F}_{t} .
$$

Then, $\tilde{\Delta} \in \mathscr{S}^{\infty}$ by Definition 2.7(ii), and

$$
E^{\mathbb{Q}}\left[\tilde{\Delta}_{t}\left(S_{t+1}-S_{t}\right) \mid \mathscr{F}_{t}\right]=\max \left\{\mathbb{E}^{\mathbb{Q}}\left[\Delta_{t} \cdot\left(S_{t+1}-S_{t}\right) \mid \mathscr{F}_{t}\right], \mathbb{E}^{\mathbb{Q}}\left[\Delta_{t}^{\prime} \cdot\left(S_{t+1}-S_{t}\right) \mid \mathscr{F}_{t}\right]\right\}
$$

We can therefore apply [21, Theorem A.33,pg. 496] and get

$$
\mathbb{E}^{\mathbb{Q}}\left[\operatorname{ess}_{\Delta \in \mathscr{S}^{\infty}} \mathbb{Q}^{\mathbb{Q}} \mathbb{E}^{\mathbb{Q}}\left[\Delta_{t} \cdot\left(S_{t+1}-S_{t}\right) \mid \mathscr{F}_{t}\right]\right]=\sup _{\Delta \in \mathscr{S}^{\infty}} \mathbb{E}^{\mathbb{Q}}\left[\Delta_{t} \cdot\left(S_{t+1}-S_{t}\right)\right] .
$$

Now, in view of (3.4), we have

$$
\mathbb{E}^{\mathbb{Q}}\left[A_{t}^{\mathbb{Q}}\right]=\sum_{i=1}^{t} \sup _{\Delta \in \mathscr{S}^{\infty}} \mathbb{E}^{\mathbb{Q}}\left[\Delta_{i-1} \cdot\left(S_{i}-S_{i-1}\right)\right]=\sup _{\Delta \in \mathscr{S}^{\infty}} \mathbb{E}^{\mathbb{Q}}\left[(\Delta \cdot S)_{t}\right],
$$

where the last equality follows from Definition 2.7(ii).

On strength of Definition 2.7(iii), we can actually replace $\mathscr{S}^{\infty}$ by $\mathscr{S}_{c}^{\infty}$ in 3.5 .

Lemma 3.3 For each $t=1, \ldots, T$,

$$
\mathbb{E}^{\mathbb{Q}}\left[A_{t}^{\mathbb{Q}}\right]=\sup _{\Delta \in \mathscr{S}_{c}^{\infty}} \mathbb{E}^{\mathbb{Q}}\left[(\Delta \cdot S)_{t}\right]
$$


Proof. In view of (3.5), it suffices to show that, for each fixed $\Delta \in \mathscr{S}^{\infty}$, there exists $\left\{\Delta^{\varepsilon}\right\}_{\varepsilon} \subset \mathscr{S}_{c}^{\infty}$ such that $\mathbb{E}^{\mathbb{Q}}\left[(\Delta \cdot S)_{T}\right]=\lim _{\varepsilon \rightarrow 0} \mathbb{E}^{\mathbb{Q}}\left[\left(\Delta^{\varepsilon} \cdot S\right)_{T}\right]$. Take $M>0$ such that $\left|\Delta_{t}\right| \leq M$ for all $t=1, \ldots, T-1$. By Definition 2.7(iii), for any $\varepsilon>0$, there exist $D_{\varepsilon}$ closed in $\mathbb{R}_{+}^{T}$ and $\Delta^{\varepsilon} \in \mathscr{S}_{c}^{\infty}$ such that $\mathbb{Q}\left(D_{\varepsilon}\right)>1-\varepsilon, \Delta^{\varepsilon}=\Delta$ on $D_{\varepsilon}$, and $\left|\Delta_{t}^{\varepsilon}\right| \leq M$ for all $t=1, \ldots, T-1$. It follows that

$$
\begin{aligned}
\left|\mathbb{E}^{\mathbb{Q}}\left[(\Delta \cdot S)_{T}\right]-\mathbb{E}^{\mathbb{Q}}\left[\left(\Delta^{\varepsilon} \cdot S\right)_{T}\right]\right| & \leq \mathbb{E}^{\mathbb{Q}}\left[\left|\left(\left(\Delta-\Delta^{\varepsilon}\right) \cdot S\right)_{T}\right| 1_{D_{\varepsilon}^{c}}\right] \\
& \leq \mathbb{E}^{\mathbb{Q}}\left[2 M \sum_{t=0}^{T-1}\left|S_{t+1}-S_{t}\right| 1_{D_{\varepsilon}^{c}}\right] .
\end{aligned}
$$

Thanks to Remark 2.1, the random variable $2 M \sum_{t=0}^{T-1}\left|S_{t+1}-S_{t}\right|$ is $\mathbb{Q}$-integrable. We can then conclude from the above inequality that $\mathbb{E}^{\mathbb{Q}}\left[\left(\Delta^{\varepsilon} \cdot S\right)_{T}\right] \rightarrow \mathbb{E}^{\mathbb{Q}}\left[(\Delta \cdot S)_{T}\right]$.

Definition 3.4 Let $\mathscr{Q}_{\mathscr{S}}$ be the collection of $\mathbb{Q} \in \Pi$ such that $\mathbb{E}^{\mathbb{Q}}\left[A_{T}^{\mathbb{Q}}\right]<\infty$.

Remark 3.5 If strategies in $\mathscr{S}$ are uniformly bounded, i.e. $\exists c>0$ such that $|\Delta| \leq c$ for all $\Delta \in \mathscr{S}$, then we deduce from (3.5) and Remark 2.1 that $\mathscr{Q}_{\mathscr{S}}=\Pi$.

Lemma 3.6 Given any $\Delta \in \mathscr{S}$, the process $(\Delta \cdot S)_{t}-A_{t}^{\mathbb{Q}}$ is a local $\mathbb{Q}$-supermartingale, for all $\mathbb{Q} \in \mathscr{Q}_{\mathscr{S}}$.

Proof. This result follows from the argument in [21, Proposition 9.18]. We present the proof here for completeness. Consider the stopping time

$$
\tau_{n}:=\inf \left\{t \geq 0:\left|\Delta_{t}\right|>n \text { or } \mathbb{E}^{\mathbb{Q}}\left[\left|S_{t+1}-S_{t}\right| \mid \mathscr{F}_{t}\right]>n\right\} \wedge T,
$$

where the conditional expectation is well-defined thanks to Remark 2.1. Given a process $V_{t}$, let us denote by $V_{t}^{n}$ the stopped process $V_{t \wedge \tau^{n}}$. Observe that

$$
\left|(\Delta \cdot S)_{t+1}^{n}-(\Delta \cdot S)_{t}^{n}\right| \leq 1_{\left\{\tau_{n} \geq t+1\right\}}\left|\Delta_{t}\right|\left|S_{t+1}-S_{t}\right| .
$$

Thanks again to Remark 2.1, this implies that $(\Delta \cdot S)_{t}^{n}$ is $\mathbb{Q}$-integrable. Moreover,

$\mathbb{E}^{\mathbb{Q}}\left[(\Delta \cdot S)_{t+1}^{n}-(\Delta \cdot S)_{t}^{n} \mid \mathscr{F}_{t}\right]=1_{\left\{\tau_{n} \geq t+1\right\}} \Delta_{t} \cdot\left(\mathbb{E}^{\mathbb{Q}}\left[S_{t+1} \mid \mathscr{F}_{t}\right]-S_{t}\right) \leq\left(A^{\mathbb{Q}}\right)_{t+1}^{n}-\left(A^{\mathbb{Q}}\right)_{t}^{n}$,

where the inequality follows from $\left(\underline{3.3}\right.$. Since $\mathbb{E}^{\mathbb{Q}}\left[A_{T}^{\mathbb{Q}}\right]<\infty$, the above inequality shows that $(\Delta \cdot S)_{t}^{n}-\left(A^{\mathbb{Q}}\right)_{t}^{n}$ is a $\mathbb{Q}$-supermartingale.

With some integrability at the terminal time $T$, the local supermartingale in the above result becomes a true supermartingale.

Lemma 3.7 Fix $\Delta \in \mathscr{S}$ and $\mathbb{Q} \in \mathscr{Q} \mathscr{S}$. If $\exists \mathbb{Q}$-integrable random variable $\varphi$ such that $(\Delta \cdot S)_{T} \geq \varphi \mathbb{Q}$-a.s., then $(\Delta \cdot S)_{t}-A_{t}^{\mathbb{Q}} \geq \mathbb{E}^{\mathbb{Q}}\left[\varphi-A_{T}^{\mathbb{Q}} \mid \mathscr{F}_{t}\right] \mathbb{Q}$-a.s. for all $t=1, \ldots, T$. This in particular implies that $(\Delta \cdot S)_{t}-A_{t}^{\mathbb{Q}}$ is a true $\mathbb{Q}$-supermartingale. 
Proof. Using the same notation as in the proof of Lemma 3.6 we know that there exist a sequence $\left\{\tau_{n}\right\}$ of stopping times such that $\tau^{n} \uparrow \infty \mathbb{Q}$-a.s. and the stopped process $(\Delta \cdot S)_{t}^{n}-\left(A^{\mathbb{Q}}\right)_{t}^{n}$ is a $\mathbb{Q}$-supermartingale, for each $n \in \mathbb{N}$. We will prove this lemma by induction. Given any $t=2, \ldots, T$ such that $(\Delta \cdot S)_{t}-A_{t}^{\mathbb{Q}} \geq \mathbb{E}^{\mathbb{Q}}\left[\varphi-A_{T}^{\mathbb{Q}} \mid \mathscr{F}_{t}\right]$, we obtain from the supermartingale property that

$$
\begin{aligned}
0 & \geq 1_{\left\{t-1<\tau_{n}\right\}} \mathbb{E}^{\mathbb{Q}}\left[\left((\Delta \cdot S)_{t}^{n}-\left(A^{\mathbb{Q}}\right)_{t}^{n}\right)-\left((\Delta \cdot S)_{t-1}^{n}-\left(A^{\mathbb{Q}}\right)_{t-1}^{n}\right) \mid \mathscr{F}_{t-1}\right] \\
& =\mathbb{E}^{\mathbb{Q}}\left[1_{\left\{t-1<\tau_{n}\right\}}\left\{\left((\Delta \cdot S)_{t}-A_{t}^{\mathbb{Q}}\right)-\left((\Delta \cdot S)_{t-1}-A_{t-1}^{\mathbb{Q}}\right)\right\} \mid \mathscr{F}_{t-1}\right] \\
& \geq \mathbb{E}^{\mathbb{Q}}\left[1_{\left\{t-1<\tau_{n}\right\}}\left\{\mathbb{E}^{\mathbb{Q}}\left[\varphi-A_{T}^{\mathbb{Q}} \mid \mathscr{F}_{t}\right]-\left((\Delta \cdot S)_{t-1}-A_{t-1}^{\mathbb{Q}}\right)\right\} \mid \mathscr{F}_{t-1}\right] \\
& =1_{\left\{t-1<\tau_{n}\right\}} \mathbb{E}^{\mathbb{Q}}\left[\varphi-A_{T}^{\mathbb{Q}} \mid \mathscr{F}_{t-1}\right]-1_{\left\{t-1<\tau_{n}\right\}}\left((\Delta \cdot S)_{t-1}-A_{t-1}^{\mathbb{Q}}\right) .
\end{aligned}
$$

Sending $n \rightarrow \infty$, we conclude that $(\Delta \cdot S)_{t-1}-A_{t-1}^{\mathbb{Q}} \geq \mathbb{E}^{\mathbb{Q}}\left[\varphi-A_{T}^{\mathbb{Q}} \mid \mathscr{F}_{t-1}\right] \mathbb{Q}$-a.s. Now, by Lemma 3.6 $(\Delta \cdot S)_{t}-A_{t}^{\mathbb{Q}}$ is a local $\mathbb{Q}$-supermartingale bounded from below by a martingale, and thus a true $\mathbb{Q}$-supermartingale (see e.g. [21, Proposition 9.6]).

3.2 Derivation of the superhedging duality

In view of the static holdings of $\left\{\psi_{i}\right\}_{i \in I}$ in 3.1 , we introduce

$$
\mathscr{E}_{I}^{\mathbb{Q}}:=\sup _{\eta \in \mathscr{R}^{I}} \sum_{i \in I}\left(\eta_{i} \mathbb{E}^{\mathbb{Q}}\left[\psi_{i}\right]-c_{i}\left(\eta_{i}\right)\right) \geq 0 \text { for } \mathbb{Q} \in \Pi \text {. }
$$

Set $F(I):=\{J \subseteq I: J$ is a finite set $\}$. We observe that

$$
\mathscr{E}_{I}^{\mathbb{Q}}=\sup _{J \in F(I)} \sup _{\eta \in \mathbb{R}^{|J|}} \sum_{i \in J}\left(\eta_{i} \mathbb{E}^{\mathbb{Q}}\left[\psi_{i}\right]-c_{i}\left(\eta_{i}\right)\right)=\sup _{J \in F(I)} \sum_{i \in J} \sup _{\eta \in \mathbb{R}}\left(\eta \mathbb{E}^{\mathbb{Q}}\left[\psi_{i}\right]-c_{i}(\eta)\right) .
$$

Consider the collection of measures

$$
\mathscr{Q}_{\mathscr{S}, I}:=\left\{\mathbb{Q} \in \mathscr{Q}_{\mathscr{S}}: \mathscr{E}_{I}^{\mathbb{Q}}<\infty\right\}
$$

Remark 3.8 Fix $\mathbb{Q} \in \Pi$. For any $i \in I$, suppose the following two conditions hold.

(i) $c_{i}(\eta)=\infty$ for some $\eta>0$ or $\mathbb{E}^{\mathbb{Q}}\left[\psi_{i}\right]<c_{i}^{\prime}(\infty)$,

(ii) $c_{i}(\eta)=\infty$ for some $\eta<0$ or $\mathbb{E}^{\mathbb{Q}}\left[\psi_{i}\right]>c_{i}^{\prime}(-\infty)$.

By the convexity of $\eta \mapsto c_{i}(\eta)$, we have $\sup _{\eta \in \mathbb{R}}\left(\eta \mathbb{E}^{\mathbb{Q}}\left[\psi_{i}\right]-c_{i}(\eta)\right)<\infty$. Thus, in view of (3.7), if I is a finite set, and (i)-(ii) above are satisfied for all $i \in I$, then $\mathscr{E}_{I}^{\mathbb{Q}}<\infty$.

We will work on deriving a duality between $D(\Phi)$ defined in (3.2) and

$$
P(\Phi):=\sup _{\mathbb{Q} \in \mathscr{Q}_{\mathscr{S}, I}}\left\{\mathbb{E}^{\mathbb{Q}}\left[\Phi-A_{T}^{\mathbb{Q}}\right]-\mathscr{E}_{I}^{\mathbb{Q}}\right\}
$$

The following minimax result, taken from [34, Corollary 2], will be useful. 
Lemma 3.9 Let $X$ be a compact convex subset of a topological vector space, $Y$ be a convex subset of a vector space, and $f: X \times Y \mapsto \mathbb{R}$ be a function satisfying

(i) For each $x \in X$, the map $y \mapsto f(x, y)$ is convex on $Y$.

(ii) For each $y \in Y$, the map $x \mapsto f(x, y)$ is upper semicontinuous and concave on $X$.

Then, $\inf _{y \in Y} \sup _{x \in X} f(x, y)=\sup _{x \in X} \inf _{y \in Y} f(x, y)$.

Let us first derive a superhedging duality for the case where $I=\emptyset$, i.e. no option is tradable at time 0 except vanilla calls. The pathwise relation in (3.1) reduces to

$$
\Psi_{u, \Delta}(x):=\sum_{t=1}^{T} \sum_{n=1}^{d} u_{t}^{n}\left(x_{t}^{n}\right)+(\Delta \cdot x)_{T} \geq \Phi(x), \forall x \in\left(\mathbb{R}_{+}^{d}\right)^{T} .
$$

By the convention that the sum over an empty set is $0, D(\Phi)$ in 3.2 becomes

$$
\begin{array}{r}
D_{\emptyset}(\Phi):=\inf \left\{\sum_{t=1}^{T} \sum_{n=1}^{d} \int_{\mathbb{R}_{+}} u_{t}^{n} d \mu_{t}^{n}: u \in \mathscr{U} \text { and } \Delta \in \mathscr{S} \text { such that } \Psi_{u, \Delta}(x) \geq \Phi(x)\right. \\
\left.\forall x \in\left(\mathbb{R}_{+}^{d}\right)^{T}\right\} .
\end{array}
$$

Also, since $I=\emptyset$ implies that $F(I)=\{\emptyset\}$, we deduce from $(3.7)$ that $\mathscr{E}_{I}^{\mathbb{Q}}=0$, as it is a summation over an empty set. It follows that $P(\Phi)$ in (3.9) reduces to

$$
P_{\emptyset}(\Phi):=\sup _{\mathbb{Q} \in \mathscr{Q}_{\mathscr{S}}} \mathbb{E}^{\mathbb{Q}}\left[\Phi-A_{T}^{\mathbb{Q}}\right]
$$

Proposition 3.10 Let $I=\emptyset$. Suppose $\Phi:\left(\mathbb{R}_{+}^{d}\right)^{T} \mapsto \mathbb{R}$ is measurable and $\exists K>0$ such that

$$
\Phi\left(x_{1}, \ldots, x_{T}\right) \leq K\left(1+\sum_{t=1}^{T} \sum_{n=1}^{d} x_{t}^{n}\right), \text { for all } x \in\left(\mathbb{R}_{+}^{d}\right)^{T} .
$$

(i) We have $P_{\emptyset}(\Phi) \leq D_{\emptyset}(\Phi)$.

(ii) If $\Phi$ is upper semicontinuous, then $P_{\emptyset}(\Phi)=D_{\emptyset}(\Phi)$.

(iii) If $\Phi$ is upper semicontinuous and $\mathscr{Q}_{\mathscr{S}} \neq \emptyset$, there exists $\mathbb{Q}^{*} \in \mathscr{Q}_{\mathscr{S}}$ such that $P_{\emptyset}(\Phi)=\mathbb{E}^{\mathbb{Q}^{*}}\left[\Phi-A_{T}^{\mathbb{Q}^{*}}\right]$

Proof. First, by Remark 2.1, 3.11, and Definition 3.4, $P_{\emptyset}$ is indeed well defined.

(i) Take $u \in \mathscr{U}$ and $\Delta \in \mathscr{S}$ such that $\Psi_{u, \Delta} \geq \Phi$. For any $\mathbb{Q} \in \mathscr{Q}_{\mathscr{S}}$, note that

$$
(\Delta \cdot S)_{T} \geq \Phi(x)-\sum_{t=1}^{T} \sum_{n=1}^{d} u_{t}^{n}\left(x_{t}^{n}\right)
$$

If $\mathbb{E}^{\mathbb{Q}}\left[\Phi^{-}\right]<\infty$, then $\Phi(x)-\sum_{t=1}^{T} \sum_{i=1}^{d} u_{t}^{i}\left(x_{t}\right)$ is $\mathbb{Q}$-integrable thanks to (3.11). We then conclude from Lemma 3.7 that $(\Delta \cdot S)_{t}-A_{t}^{\mathbb{Q}}$ is a true $\mathbb{Q}$-supermartingale. Hence,

$$
\mathbb{E}^{\mathbb{Q}}\left[\Phi-A_{T}^{\mathbb{Q}}\right] \leq \mathbb{E}^{\mathbb{Q}}\left[\sum_{t=1}^{T} \sum_{n=1}^{d} u_{t}^{n}\left(S_{t}^{n}\right)+(\Delta \cdot S)_{T}-A_{T}^{\mathbb{Q}}\right] \leq \sum_{t=1}^{T} \sum_{n=1}^{d} \int_{\mathbb{R}_{+}} u_{t}^{n} d \mu_{t}^{n}
$$


If $\mathbb{E}^{\mathbb{Q}}\left[\Phi^{-}\right]=\infty$, then (3.12) trivially holds. By taking supremum over $\mathbb{Q} \in \mathscr{Q}_{\mathscr{S}}$ and using the arbitrariness of $u$, we obtain from (3.12) the desired inequality.

(ii) We will use an argument similar to [5, equations (3.1)-(3.4)]. First, observe that

$$
\begin{aligned}
& D_{\emptyset}(\Phi) \leq \inf \left\{\sum_{t=1}^{T} \sum_{n=1}^{d} \int_{\mathbb{R}_{+}} u_{t}^{n} d \mu_{t}^{i}: u \in \mathscr{U} \text { and } \Delta \in \mathscr{S}_{c}^{\infty} \text { such that } \Psi_{u, \Delta}(x) \geq \Phi(x)\right\} \\
& =\inf _{\Delta \in \mathscr{S}_{c}^{\infty}} \inf \left\{\sum_{t=1}^{T} \sum_{n=1}^{d} \int_{\mathbb{R}_{+}} u_{t}^{n} d \mu_{t}^{n}: u \in \mathscr{U} \text { such that } \sum_{t=1}^{T} \sum_{n=1}^{d} u_{t}^{n}\left(x_{t}^{n}\right) \geq \Phi(x)-(\Delta \cdot x)_{T}\right\} \\
& =\inf _{\Delta \in \mathscr{S}_{c}^{\infty}} \sup _{\mathbb{Q} \in \Pi} \mathbb{E}^{\mathbb{Q}}\left[\Phi(x)-(\Delta \cdot x)_{T}\right] .
\end{aligned}
$$

Here, (3.13) follows from the theory of optimal transport (see e.g. [5], Proposition $2.1]$ ), which requires the upper semicontinuity of $\Phi$. Now, we intend to apply Lemma 3.9 to 3.13, with $X=\Pi, Y=\mathscr{S}_{c}^{\infty}$, and $f(\mathbb{Q}, \Delta)=\mathbb{E}^{\mathbb{Q}}\left[\Phi(x)-(\Delta \cdot x)_{T}\right]$. The only condition in Lemma 3.9 which is not obvious is the upper semicontinuity of $\mathbb{Q} \mapsto f(\mathbb{Q}, \Delta)$. For each $\Delta \in \mathscr{S}_{c}^{\infty}$, thanks to (3.11), the upper semicontinuous function $\Phi(x)-(\Delta \cdot x)_{T}$ is bounded from above by the continuous function

$$
\ell(x):=K\left(1+\sum_{t=1}^{T} \sum_{n=1}^{d} x_{t}^{n}\right)+|\Delta|_{\infty} \sum_{n=1}^{d}\left(x_{0}^{n}+2\left(x_{1}^{n}+\cdots+x_{T-1}^{n}\right)+x_{T}^{n}\right),
$$

where $|\Delta|_{\infty}:=\left|\Delta_{0}\right| \vee \max \left\{\sup _{z \in\left(\mathbb{R}_{+}^{d}\right)^{t}}\left|\Delta_{t}(z)\right|: t=1, \ldots, T-1\right\}<\infty$. Take any sequence $\left\{\mathbb{Q}_{n}\right\}_{n \in \mathbb{N}}$ in $\Pi$ which converge weakly to some $\mathbb{Q}^{*} \in \Pi$. Observing that $\mathbb{Q} \mapsto \mathbb{E}^{\mathbb{Q}}[\ell]$ is a constant function on $\Pi$, we conclude from [35, Lemma 4.3] that

$$
\underset{n \rightarrow \infty}{\limsup } \mathbb{E}^{\mathbb{Q}_{n}}\left[\Phi(x)-(\Delta \cdot x)_{T}\right] \leq \mathbb{E}^{\mathbb{Q}^{*}}\left[\Phi(x)-(\Delta \cdot x)_{T}\right],
$$

which shows the upper semicontinuity of $\mathbb{Q} \mapsto f(\mathbb{Q}, \Delta)$. Now, applying Lemma 3.9 to 3.13 yields

$$
\begin{aligned}
D_{\emptyset}(\Phi) & \leq \sup _{\mathbb{Q} \in \Pi} \inf _{\Delta \in \mathscr{S}_{c}^{\infty}} \mathbb{E}^{\mathbb{Q}}\left[\Phi(x)-(\Delta \cdot x)_{T}\right]=\sup _{\mathbb{Q} \in \Pi}\left\{\mathbb{E}^{\mathbb{Q}}[\Phi]-\sup _{\Delta \in \mathscr{S}_{c}^{\infty}} \mathbb{E}^{\mathbb{Q}}\left[(\Delta \cdot S)_{T}\right]\right\} \\
& =\sup _{\mathbb{Q} \in \Pi}\left\{\mathbb{E}^{\mathbb{Q}}[\Phi]-\mathbb{E}^{\mathbb{Q}}\left[A_{T}^{\mathbb{Q}}\right]\right\}=\sup _{\mathbb{Q} \in \mathscr{Q} \mathscr{S}}\left\{\mathbb{E}^{\mathbb{Q}}[\Phi]-\mathbb{E}^{\mathbb{Q}}\left[A_{T}^{\mathbb{Q}}\right]\right\}=P_{\emptyset}(\Phi),
\end{aligned}
$$

where the second line follows from Lemma 3.3 .

(iii) In view of Definition 3.4, we can write $P_{\emptyset}(\Phi)=\sup _{\mathbb{Q} \in \Pi} \mathbb{E}^{\mathbb{Q}}\left[\Phi-A_{T}^{\mathbb{Q}}\right]$ by replacing $\mathscr{Q}_{\mathscr{S}}$ by $\Pi$ in $(3.10)$. Since $\Pi$ is compact under the topology of weak convergence (Remark 2.2), it suffices to show that $\mathbb{Q} \mapsto f(\mathbb{Q}):=\mathbb{E}^{\mathbb{Q}}\left[\Phi-A_{T}^{\mathbb{Q}}\right]$ is upper semicontinuous. Since the argument in part (ii) already implies that $\mathbb{Q} \mapsto \mathbb{E}^{\mathbb{Q}}[\Phi]$ is upper semicontinuous, it remains to show that $\mathbb{Q} \mapsto g(\mathbb{Q}):=\mathbb{E}^{\mathbb{Q}}\left[A_{T}^{\mathbb{Q}}\right]$ is lower semicontinuous. Similar to (3.14), for each $\Delta \in \mathscr{S}_{c}^{\infty}$, we have $\left|(\Delta \bullet x)_{T}\right| \leq h(x)$ with $h$ defined by $h(x):=|\Delta|_{\infty} \sum_{n=1}^{d}\left(x_{0}^{n}+2\left(x_{1}^{n}+\cdots+x_{T-1}^{n}\right)+x_{T}^{n}\right]$. For any sequence $\left\{\mathbb{Q}_{n}\right\}_{n \in \mathbb{N}}$ in $\Pi$ which converge weakly to some $\mathbb{Q}^{*} \in \Pi$, applying [35, Lemma 4.3] to the functions $(\Delta \cdot x)_{T}$ and $-(\Delta \cdot x)_{T}$ gives

$$
\liminf _{n \rightarrow \infty} \mathbb{E}^{\mathbb{Q}_{n}}\left[(\Delta \cdot x)_{T}\right] \geq \mathbb{E}^{\mathbb{Q}^{*}}\left[(\Delta \cdot x)_{T}\right] \geq \limsup _{n \rightarrow \infty} \mathbb{E}^{\mathbb{Q}_{n}}\left[(\Delta \cdot x)_{T}\right]
$$


It follows that $\mathbb{Q} \mapsto g_{\Delta}(\mathbb{Q}):=\mathbb{E}^{\mathbb{Q}}\left[(\Delta \bullet S)_{T}\right]$ is continuous. Thanks to Lemma 3.3, we have $g(\mathbb{Q})=\sup _{\Delta \in \mathscr{S}_{c}^{\infty}} g_{\Delta}(\mathbb{Q})$ is lower semicontinuous, as a supremum of continuous functions.

Remark 3.11 The condition $\mathscr{Q}_{\mathscr{S}} \neq \emptyset$ is not needed for Proposition 3.10 (i) and (ii). Indeed, if $\mathscr{Q}_{\mathscr{S}}=\emptyset$, then $P(\Phi)=-\infty$ and thus part (i) trivially holds; also, the arguments in part (ii) hold true as long as $\Pi \neq \emptyset$, which is guaranteed by Remark 2.2

Remark 3.12 Proposition 3.10 extends [5. Theorem 1.1] to the case with portfolio constraints. To see this, consider the no-constraint case, i.e. $\mathscr{S}=\mathscr{H}$. Observe that $\mathscr{S}=\mathscr{H}$ implies $\mathscr{Q}_{\mathscr{S}}=\mathscr{M}$, with $\mathscr{M}$ defined as in (2.3). While $\mathscr{M} \subseteq \mathscr{Q}_{\mathscr{S}}$ is obvious, the other inclusion follows from Definition 3.1 Indeed, given $\mathbb{Q} \in \mathscr{Q}_{\mathscr{S}} \backslash \mathscr{M}$, there must exist $t \in\{0, \ldots, T-1\}$ such that $\mathbb{E}^{\mathbb{Q}}\left[S_{t+1} \mid \mathscr{F}_{t}\right] \neq S_{t}$. Since $\mathscr{S}=\mathscr{H}$, we have $A_{t+1}^{\mathbb{Q}}-A_{t}^{\mathbb{Q}}=\infty$, contradicting $\mathbb{Q} \in \mathscr{Q}_{\mathscr{S}}$. The duality in Proposition 3.10 reduces to

$$
D_{\emptyset}(\Phi)=P_{\emptyset}(\Phi)=\sup _{\mathbb{Q} \in \mathscr{M}} \mathbb{E}^{\mathbb{Q}}[\Phi]
$$

which recovers [5] Theorem 1.1].

Remark 3.13 Proposition 3.10 also extends [5. Theorem 1.1] to the case with multidimensional S. Since [5. Theorem 1.1] relies on one-dimensional Monge-Kantorovich duality which works on the product of $T$ copies of $\mathbb{R}_{+}$(i.e. [5. Proposition 2.1]), one may expect to prove Proposition 3.10 via multi-dimensional Monge-Kantorovich duality which works on the product of $T$ copies of $\mathbb{R}_{+}^{d}$. While such a duality does exist (e.g. [29. Theorem 2.14]), applying it requires the knowledge of the joint distribution of $\left(S_{t}^{1}, \ldots, S_{t}^{d}\right)$ for each $t=1, \ldots, T$. This is not practically feasible, as vanilla calls only specify the distribution of $S_{t}^{n}$, for each $n$ and $t$.

As a result, in Proposition 3.10 we still rely on the one-dimensional result [5. Proposition 2.1]. By treating $\Omega=\left(\mathbb{R}_{+}^{d}\right)^{T}$ as the product of $(d \times T)$ copies of $\mathbb{R}_{+}$(as in Remark 2.2], [5. Proposition 2.1] is indeed applicable as the distribution $\mu_{t}^{n}$ of $S_{t}^{n}$, for each $n=1, \ldots, d$ and $t=1, \ldots, T$, is known. Note that it was first mentioned in [23. Theorem 2.1] that [5. Theorem 1.1] could be generalized to higher dimensions.

By the convexity of $c_{i}$, Proposition 3.10 extends to the general case where $I \neq \emptyset$.

Theorem 3.14 Suppose $\psi_{i}$ is continuous and $\left|\psi_{i}\right|$ satisfies (3.11) for all $i \in I$. Then, for any upper semicontinuous function $\Phi:\left(\mathbb{R}_{+}^{d}\right)^{T} \mapsto \mathbb{R}$ satisfying (3.11), we have $D(\Phi)=P(\Phi)$, with $D$ and $P$ defined as in (3.2) and (3.9). Moreover, if $\mathscr{Q}_{\mathscr{S}, I} \neq \emptyset$, the supremum in (3.9) is attained at some $\mathbb{Q}^{*} \in \mathscr{Q}_{\mathscr{S}, I}$.

Proof. Observe from (3.2) and Proposition 3.10 that

$$
\begin{aligned}
D(\Phi) & =\inf _{\eta \in \mathscr{R}^{I}} D_{\emptyset}\left(\Phi-\sum_{i \in I}\left(\eta_{i} \psi_{i}-c_{i}\left(\eta_{i}\right)\right)\right) \\
& =\inf _{\eta \in \mathscr{R}^{I}} \sup _{\mathbb{Q} \in \Pi} \mathbb{E}^{\mathbb{Q}}\left[\Phi-\sum_{i \in I}\left(\eta_{i} \psi_{i}-c_{i}\left(\eta_{i}\right)\right)-A_{T}^{\mathbb{Q}}\right] .
\end{aligned}
$$


Consider the function $f(\mathbb{Q}, \eta):=\mathbb{E}^{\mathbb{Q}}\left[\Phi-\sum_{i \in I}\left(\eta_{i} \psi_{i}-c_{i}\left(\eta_{i}\right)\right)-A_{T}^{\mathbb{Q}}\right]$ for $\mathbb{Q} \in \Pi$ and $\eta \in \mathscr{R}^{I}$. By the upper semicontinuity of $\Phi$, the continuity of $\psi_{i}$, and $(\underline{3.11})$, we may argue as in Proposition 3.10 (i) and (ii) that $f$ is upper semicontinuous in $\mathbb{Q} \in \Pi$. Moreover, by the convexity of $\eta \mapsto c_{i}(\eta)$ for all $i \in I, f$ is convex in $\eta \in \mathscr{R}^{I}$. Thus, we may apply Lemma 3.9 to 3.15) and get

$$
\begin{aligned}
D(\Phi) & =\sup _{\mathbb{Q} \in \Pi} \inf _{\eta \in \mathscr{R}^{I}} \mathbb{E}^{\mathbb{Q}}\left[\Phi-\sum_{i \in I}\left(\eta_{i} \psi_{i}-c_{i}\left(\eta_{i}\right)\right)-A_{T}^{\mathbb{Q}}\right] \\
& =\sup _{\mathbb{Q} \in \mathscr{Q}_{\mathscr{S}, I}}\left\{\mathbb{E}^{\mathbb{Q}}\left[\Phi-A_{T}^{\mathbb{Q}}\right]-\mathscr{E}_{I}^{\mathbb{Q}}\right\}=P(\Phi) .
\end{aligned}
$$

In view of the argument in Proposition 3.10 (iii) and the continuity of $\psi_{i}$, we obtain that $\mathbb{Q} \mapsto \mathbb{E}^{\mathbb{Q}}\left[\Phi-A_{T}^{\mathbb{Q}}\right]-\mathscr{E}_{I}^{\mathbb{Q}}$ is upper semicontinuous on the compact set $\Pi$. Thus, the supremum in (3.9) is attained if $\mathscr{Q}_{\mathscr{S}, I} \neq \emptyset$.

\subsection{Connection to the model-free duality in [1]}

Consider the case where every option $\psi_{i}$ can actually be liquidly traded at time 0 , just as vanilla calls. That is, for each $i \in I, c_{i}$ is linear (see Remark 2.5), and we let $p_{i} \in \mathbb{R}$ be the slope of $c_{i}$. By $(3.6), \mathscr{E}_{I}^{\mathbb{Q}}$ equals 0 iff $\mathbb{E}^{\mathbb{Q}}\left[\psi_{i}\right]=p_{i}$ for all $i \in I$, and $\infty$ if otherwise. It follows from 3.8 that

$$
\mathscr{Q}_{\mathscr{S}, I}=\mathscr{Q}_{\mathscr{S},\left(p_{i}\right)_{i \in I}}:=\left\{\mathbb{Q} \in \mathscr{Q}_{\mathscr{S}}: \mathbb{E}^{\mathbb{Q}}\left[\psi_{i}\right]=p_{i}, \forall i \in I\right\}
$$

Recall $\mathscr{M}$ defined in 2.3. Let us also consider

$$
\mathscr{M}_{I}:=\left\{\mathbb{Q} \in \mathscr{M}: c_{i}^{\prime}(0-) \leq \mathbb{E}^{\mathbb{Q}}\left[\psi_{i}\right] \leq c_{i}^{\prime}(0+), \forall i \in I\right\} .
$$

Under current setting, it becomes

$$
\mathscr{M}_{I}=\mathscr{M}_{\left(p_{i}\right)_{i \in I}}:=\left\{\mathbb{Q} \in \mathscr{M}: \mathbb{E}^{\mathbb{Q}}\left[\psi_{i}\right]=p_{i}, \forall i \in I\right\} .
$$

Corollary 3.15 For each $i \in I$, suppose $\psi_{i}$ is continuous, $\left|\psi_{i}\right|$ satisfies $\left(3.11\right.$, and $\psi_{i}$ can be traded liquidly at the price $p_{i} \in \mathbb{R}$. Let $\Phi:\left(\mathbb{R}_{+}^{d}\right)^{T} \mapsto \mathbb{R}$ be upper semicontinuous and satisfy 3.11.

(i) We have

$$
D(\Phi)=P(\Phi)=\sup _{\mathbb{Q} \in \mathscr{Q}_{\mathscr{S},\left(P_{i}\right)_{i \in I}}} \mathbb{E}^{\mathbb{Q}}\left[\Phi-A_{T}^{\mathbb{Q}}\right] .
$$

(ii) Furthermore, if there is no portfolio constraint, i.e. $\mathscr{S}=\mathscr{H}$, then

$$
D(\Phi)=P(\Phi)=\sup _{\mathbb{Q} \in \mathscr{M}_{\left(p_{i}\right)_{i \in I}}} \mathbb{E}^{\mathbb{Q}}[\Phi] .
$$

Proof. (i) simply follows from Theorem 3.14 and 3.16). For (ii), recalling from Remark 3.12 that $\mathscr{S}=\mathscr{H}$ implies $\mathscr{Q}_{\mathscr{S}}=\mathscr{M}$, we have $\mathscr{Q}_{\mathscr{S},\left(p_{i}\right)_{i \in I}}=\mathscr{M}_{\left(p_{i}\right)_{i \in I}}$. Then, part (i) just becomes the desired result. 
Remark 3.16 Corollary 3.15 (ii) states that to find the superhedging price of $\Phi$, one needs to consider expectations of $\Phi$ under martingale measures which are consistent with market prices of both vanilla calls and other options $\left\{\psi_{i}\right\}_{i \in I}$. This in particular recovers [1] Theorem 1.4], for the case where tradable options at time 0 include at least vanilla calls with all maturities and strikes.

\subsection{Connection to convex risk measures}

Let $\mathscr{X}$ be the collection of measurable functions $\Phi:\left(\mathbb{R}_{+}^{d}\right)^{T} \mapsto \mathbb{R}$ satisfying the linear growth condition (3.11). We say $\rho: \mathscr{X} \mapsto \mathbb{R}$ is a convex risk measure if for all $\Phi$, $\Phi^{\prime} \in \mathscr{X}$, the following conditions hold:

- Monotonicity: If $\Phi \leq \Phi^{\prime}$, then $\rho(\Phi) \geq \rho\left(\Phi^{\prime}\right)$.

- Translation Invariance: If $m \in \mathbb{R}$, then $\rho(\Phi+m)=\rho(\Phi)-m$.

- Convexity: If $0 \leq \lambda \leq 1$, then $\rho\left(\lambda \Phi+(1-\lambda) \Phi^{\prime}\right) \leq \lambda \rho(\Phi)+(1-\lambda) \rho\left(\Phi^{\prime}\right)$.

Consider the acceptance set

$\mathscr{A}_{\mathscr{S}}:=\left\{\Phi \in \mathscr{X}: u \in \mathscr{U}_{0}, \eta \in \mathscr{R}^{I}\right.$, and $\Delta \in \mathscr{S}$ such that

$$
\left.\Phi(x)+\Psi_{u, \eta, \Delta}(x) \geq 0, \forall x \in\left(\mathbb{R}^{d}\right)^{T}\right\} .
$$

Then, define the function $\rho_{\mathscr{S}}: \mathscr{X} \mapsto \mathbb{R}$ by

$$
\rho_{\mathscr{S}}(\Phi):=\inf \left\{m \in \mathbb{R}: m+\Phi \in \mathscr{A}_{\mathscr{S}}\right\}=D(-\Phi) .
$$

Proposition 3.17 If $\mathscr{Q}_{\mathscr{S}, I} \neq \emptyset$, then $\rho_{\mathscr{S}}$ is a convex risk measure, and admits the dual formulation

$$
\rho_{\mathscr{S}}(\Phi)=\sup _{\mathbb{Q} \in \Pi}\left(\mathbb{E}^{\mathbb{Q}}[-\Phi]-\alpha^{*}(\mathbb{Q})\right),
$$

where the penalty function $\alpha^{*}$ is given by

$$
\alpha^{*}(\mathbb{Q}):= \begin{cases}\mathbb{E}^{\mathbb{Q}}\left[A_{T}^{\mathbb{Q}}\right]+\mathscr{E}_{I}^{\mathbb{Q}} & \text { if } \mathbb{Q} \in \mathscr{Q}_{\mathscr{S}, I}, \\ \infty, & \text { otherwise. }\end{cases}
$$

Moreover, for any $\alpha: \Pi \mapsto \mathbb{R} \cup\{\infty\}$ such that (3.19) holds (with $\alpha^{*}$ replaced by $\alpha$ ), we have $\alpha^{*}(\mathbb{Q}) \leq \alpha(\mathbb{Q})$ for all $\mathbb{Q} \in \Pi$.

Proof. Monotonicity and translation invariance can be easily verified, while the convexity of $\rho_{\mathscr{S}}$ follows from the convexity of $\mathscr{U}_{0}, \mathscr{R}^{I}$ and $\mathscr{S}$. Now, the duality (3.19) is a direct consequence of Theorem 3.14. Since $\mathscr{Q}_{\mathscr{S}, I} \neq \emptyset$, (3.19) shows that $\rho_{\mathscr{S}}$ is real-valued, and thus a convex risk measure. To show that $\alpha^{*}$ is the minimal penalty function, observe that for any $\alpha: \Pi \mapsto \mathbb{R} \cup\{\infty\}$ satisfying [3.19], we have

$$
\begin{aligned}
\alpha(\mathbb{Q}) \geq \sup _{\Phi \in \mathscr{X}}\left(\mathbb{E}^{\mathbb{Q}}[-\Phi]-\rho_{\mathscr{S}}(\Phi)\right) & \geq \sup _{\Phi \in \mathscr{A}_{\mathscr{S}}}\left(\mathbb{E}^{\mathbb{Q}}[-\Phi]-\rho_{\mathscr{S}}(\Phi)\right) \\
& \geq \sup _{\Phi \in \mathscr{A}_{\mathscr{S}}} \mathbb{E}^{\mathbb{Q}}[-\Phi], \forall \mathbb{Q} \in \Pi .
\end{aligned}
$$


By Lemma 3.3 and (3.6),

$$
\alpha^{*}(\mathbb{Q})=\sup \left\{\mathbb{E}^{\mathbb{Q}}\left[(\Delta \cdot S)_{T}+\sum_{i \in I}\left(\eta_{i} \psi_{i}-c_{i}\left(\eta_{i}\right)\right)\right]: \Delta \in \mathscr{S}^{\infty}, \eta \in \mathscr{R}^{I}\right\}
$$

for all $\mathbb{Q} \in \Pi$. Since $-(\Delta \cdot S)_{T}-\sum_{i \in I}\left(\eta_{i} \psi_{i}-c_{i}\left(\eta_{i}\right)\right) \in \mathscr{A}_{\mathscr{S}}$ for all $\Delta \in \mathscr{S}^{\infty}$ and $\eta \in \mathscr{R}^{I}$, we conclude from $(3.20)$ that $\alpha^{*}(\mathbb{Q}) \leq \alpha(\mathbb{Q})$.

Remark 3.18 Proposition 3.17 generalizes Proposition 16 and Theorem 17 in [20] to a model-independent setting. Note that a no-arbitrage condition (under a given physical measure $\mathbb{P}$ ) is imposed in [20]. Here, we require only $\mathscr{Q}_{\mathscr{S} . I} \neq \emptyset$, which is weaker than the model-independent no-arbitrage condition; see Section 4 for details.

\section{Fundamental theorem of asset pricing via duality}

Following the formulation in [1], we introduce the notion of arbitrage in the strong pathwise sense:

Definition 4.1 (model-independent arbitrage) We say there is model-independent arbitrage under the constraint $\mathscr{S}$, if there exist $u \in \mathscr{U}_{0}, \eta \in \mathscr{R}^{I}$, and $\Delta \in \mathscr{S}$ such that

$$
\sum_{t=1}^{T} \sum_{n=1}^{d} u_{t}^{n}\left(x_{t}^{n}\right)+\sum_{i \in I}\left(\eta_{i} \psi_{i}(x)-c_{i}\left(\eta_{i}\right)\right)+(\Delta \cdot x)_{T}>0, \text { for all } x \in\left(\mathbb{R}_{+}^{d}\right)^{T} .
$$

Remark 4.2 It is immediate from the above definition that if model-independent arbitrage exists, then it is arbitrage under any probability measure $\mathbb{P}$ defined on $\Omega$.

Note that instead of using the pathwise formulation, the authors in [7] introduce a weaker notion of arbitrage under model uncertainty via quasi-sure analysis. They include more strategies in the definition of arbitrage, and provide different characterization of no-arbitrage condition and superhedging duality. We, however, will not pursue this direction in this paper.

Consider the following collection of measure

$\mathscr{P}_{\mathscr{S}}:=\left\{\mathbb{Q} \in \Pi:\left\{(\Delta \cdot S)_{t}\right\}_{t=0}^{T}\right.$ is a local $\mathbb{Q}$-supermartingale, for all $\left.\Delta \in \mathscr{S}\right\}$.

Remark $4.3\left(\mathscr{M}\right.$ and $\left.\mathscr{P}_{\mathscr{S}}\right)$ By definition, we see that $\mathscr{M} \subset \mathscr{P}_{\mathscr{S}}$. Given $\alpha>0$, if $\bar{\alpha}:=\left\{\Delta_{t}^{n} \equiv \alpha\right\}_{t, n}$ and $-\bar{\alpha}:=\left\{\Delta_{t}^{n} \equiv-\alpha\right\}_{t, n}$ both belong to $\mathscr{S}$, then $\mathscr{P}_{\mathscr{S}}=\mathscr{M}$. Indeed, given $\mathbb{Q} \in \mathscr{P}_{\mathscr{S}}$, since $\bar{\alpha},-\bar{\alpha} \in \mathscr{S}^{\infty}, \alpha S_{t}=(\bar{\alpha} \cdot S)_{t}$ and $-\alpha S_{t}=(-\bar{\alpha} \cdot S)_{t}$ are both supermartingales under $\mathbb{Q}$. We thus conclude that $\mathbb{Q} \in \mathscr{M}$.

The following lemma provides with a characterization of $\mathscr{P}_{\mathscr{S}}$.

Lemma 4.4 Fix $\mathbb{Q} \in \Pi$. Then, $\mathbb{Q} \in \mathscr{P}_{\mathscr{S}} \Longleftrightarrow A_{T}^{\mathbb{Q}}=0 \mathbb{Q}$-a.s.

Proof. This is a consequence of [21, Proposition 9.6] and Lemma 3.6 
Remark $4.5\left(\mathscr{P}_{\mathscr{S}}\right.$ and $\left.\mathscr{Q}_{\mathscr{S}}\right)$ Lemma 4.4 in particular implies that $\mathscr{P}_{\mathscr{S}} \subseteq \mathscr{Q}_{\mathscr{S}} \mathrm{Ob}$ serve that if $\mathscr{S}^{\infty}$ is composed of all nonnegative bounded trading strategies in $\mathscr{H}$, then $\mathscr{P}_{\mathscr{S}}=\mathscr{Q}_{\mathscr{S}}$. Indeed, for any $\mathbb{Q} \in \mathscr{Q}_{\mathscr{S}}$, we see from $(3.5)$ that $A_{T}^{\mathbb{Q}}=0 \mathbb{Q}$-a.s. Then $\mathbb{Q} \in \mathscr{P}_{\mathscr{S}}$ by Lemma 4.4

To state an equivalent condition for no-arbitrage, we consider

$$
\mathscr{P}_{\mathscr{S}, I}:=\left\{\mathbb{Q} \in \mathscr{P}_{\mathscr{S}}: c_{i}^{\prime}(0-) \leq \mathbb{E}^{\mathbb{Q}}\left[\psi_{i}\right] \leq c_{i}^{\prime}(0+) \text {, for all } i \in I\right\} .
$$

Recall $\mathscr{E}_{I}^{\mathbb{Q}}$ in $\left(3.6\right.$. It is easy to verify the following characterization for $\mathscr{E}_{I}^{\mathbb{Q}}=0$.

Lemma 4.6 Given $\mathbb{Q} \in \Pi$, we have $c_{i}^{\prime}(0-) \leq \mathbb{E}^{\mathbb{Q}}\left[\psi_{i}\right] \leq c_{i}^{\prime}(0+)$ for all $i \in I$ if and ony if $\mathscr{E}_{I} \mathbb{Q}=0$.

To derive a model-independent FTAP, we need the following lemma.

Lemma 4.7 Suppose $\psi_{i}$ is continuous and $\left|\psi_{i}\right|$ satisfies (3.11) for all $i \in I$. Then,

$$
\mathscr{P}_{\mathscr{S}, I}=\emptyset \Longrightarrow \inf _{\mathbb{Q} \in \Pi}\left\{\mathbb{E}^{\mathbb{Q}}\left[A_{T}^{\mathbb{Q}}\right]+\mathscr{E}_{I}^{\mathbb{Q}}\right\}>0
$$

Proof. Assume to the contrary that $\inf _{\mathbb{Q} \in \Pi}\left\{\mathbb{E}^{\mathbb{Q}}\left[A_{T}^{\mathbb{Q}}\right]+\mathscr{E}_{I}^{\mathbb{Q}}\right\}=0$. For any $\varepsilon>0$, there exists $\mathbb{Q}_{\varepsilon} \in \Pi$ such that $0 \leq \mathbb{E}^{\mathbb{Q}_{\varepsilon}}\left[A_{T}^{\mathbb{Q}_{\varepsilon}}\right]+\mathscr{E}_{I}^{\mathbb{Q}_{\varepsilon}}<\varepsilon$. Since $\Pi$ is weakly compact (Remark 2.2), $\mathbb{Q}_{\varepsilon}$ must converge weakly to some $\mathbb{Q}^{*} \in \Pi$. For each $\Delta \in \mathscr{S}_{c}^{\infty}$, we can argue as in Proposition 3.10 (iii) to show that $\mathbb{Q} \mapsto \mathbb{E}^{\mathbb{Q}}\left[(\Delta \cdot x)_{T}\right]$ is continuous on $\Pi$ under the topology of weak convergence. Also, for each $i \in I$, since $\psi_{i}$ is continuous and $\left|\psi_{i}\right|$ satisfies (3.11), we may argue as in Proposition 3.10 (ii) to show that $\mathbb{Q} \mapsto \mathbb{E}^{\mathbb{Q}}\left[\psi_{i}\right]$ is continuous on $\Pi$. Now, by using Lemma 3.3 ,

$$
\begin{aligned}
0 & =\lim _{\varepsilon \rightarrow 0} \mathbb{E}^{\mathbb{Q}_{\varepsilon}}\left[A_{T}^{\mathbb{Q}_{\varepsilon}}\right]+\mathscr{E}_{I}^{\mathbb{Q}_{\varepsilon}}=\lim _{\varepsilon \rightarrow 0} \sup _{\Delta \in \mathscr{S}_{c}^{\infty}} \mathbb{E}^{\mathbb{Q}_{\varepsilon}}\left[(\Delta \cdot S)_{T}\right]+\sup _{\eta \in \mathscr{R}} \sum_{i \in I}\left(\eta_{i} \mathbb{E}^{\mathbb{Q}_{\varepsilon}}\left[\psi_{i}\right]-c_{i}\left(\eta_{i}\right)\right) \\
& \geq \sup _{\Delta \in \mathscr{S}_{c}^{\infty}} \lim _{\varepsilon \rightarrow 0} \mathbb{E}^{\mathbb{Q}_{\varepsilon}}\left[(\Delta \cdot S)_{T}\right]+\sup _{\eta \in \mathscr{R}^{I}} \lim _{\varepsilon \rightarrow 0} \sum_{i \in I}\left(\eta_{i} \mathbb{E}^{\mathbb{Q}_{\varepsilon}}\left[\psi_{i}\right]-c_{i}\left(\eta_{i}\right)\right) \\
& =\sup _{\Delta \in \mathscr{S}_{c}^{\infty}} \mathbb{E}^{\mathbb{Q}^{*}}\left[(\Delta \cdot S)_{T}\right]+\sup _{\eta \in \mathscr{R}^{I}} \sum_{i \in I}\left(\eta_{i} \mathbb{E}^{\mathbb{Q}^{*}}\left[\psi_{i}\right]-c_{i}\left(\eta_{i}\right)\right)=\mathbb{E}^{\mathbb{Q}^{*}}\left[A_{T}^{\mathbb{Q}^{*}}\right]+\mathscr{E}_{I} \mathbb{Q}^{*}
\end{aligned}
$$

Thus, $\mathbb{E}^{\mathbb{Q}^{*}}\left[A_{T}^{\mathbb{Q}^{*}}\right]=\mathscr{E}_{I} \mathbb{Q}^{*}=0$. By Lemmas 4.4 and 4.6, we have $\mathbb{Q}^{*} \in \mathscr{P}_{\mathscr{S}, I}$, contradicting $\mathscr{P}_{\mathscr{S}, I}=\emptyset$.

Now, we are ready to present the main result of this section.

Theorem 4.8 Suppose $\psi_{i}$ is continuous and $\left|\psi_{i}\right|$ satisfies (3.11) for all $i \in I$. Then, there is no model-independent arbitrage under constraint $\mathscr{S}$ if and only if $\mathscr{P}_{\mathscr{S}, I} \neq \emptyset$.

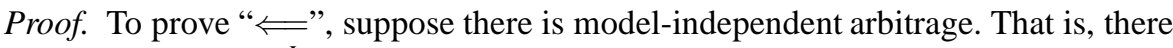
exist $u \in \mathscr{U}_{0}, \eta \in \mathscr{R}^{I}$, and $\Delta \in \mathscr{S}$ such that

$$
\sum_{t=1}^{T} \sum_{n=1}^{d} u_{t}^{n}\left(x_{t}^{n}\right)+\sum_{i \in I}\left(\eta_{i} \psi_{i}(x)-c_{i}\left(\eta_{i}\right)\right)+(\Delta \cdot x)_{T}>0 \text { for all } x \in \mathbb{R}_{+}^{T} .
$$


It follows that for any $\mathbb{Q} \in \mathscr{Q}_{\mathscr{S}, I}$,

$$
\sum_{t=1}^{T} \sum_{n=1}^{d} u_{t}^{n}\left(S_{t}^{n}\right)+\sum_{i \in I}\left(\eta_{i} \psi_{i}(S)-c_{i}\left(\eta_{i}\right)\right)+(\Delta \cdot S)_{T}-A_{T}^{\mathbb{Q}}>-A_{T}^{\mathbb{Q}} \quad \mathbb{Q} \text {-a.s. }
$$

By taking expectation on both sides, we obtain from Lemmas 3.7 that

$$
\mathscr{E}_{I}^{\mathbb{Q}} \geq \sum_{i \in I}\left(\eta_{i} \mathbb{E}^{\mathbb{Q}}\left[\psi_{i}\right]-c_{i}\left(\eta_{i}\right)\right)>-\mathbb{E}^{\mathbb{Q}}\left[A_{T}^{\mathbb{Q}}\right], \quad \text { for all } \mathbb{Q} \in \mathscr{Q}_{\mathscr{S}, I}
$$

If $\mathbb{Q} \in \mathscr{P}_{\mathscr{S}, I}$, then the above inequality becomes $\mathscr{E}_{I}^{\mathbb{Q}}>0$, thanks to Lemma 4.4 However, in view of Lemma 4.6 , this implies $\mathbb{E}^{\mathbb{Q}}\left[\psi_{i}\right] \notin\left[c_{i}^{\prime}(0-), c_{i}^{\prime}(0+)\right]$ for some $i \in I$ and thus $\mathbb{Q} \notin \mathscr{P}_{\mathscr{S}, I}$, a contradiction. Hence, we conclude that $\mathscr{P}_{\mathscr{S}, I}=\emptyset$.

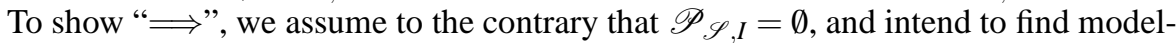
independent arbitrage. By Theorem 3.14 and Lemma 4.7, we have

$$
D(0)=\sup _{\mathbb{Q} \in \mathscr{Q}_{\mathscr{S}, I}}\left\{\mathbb{E}^{\mathbb{Q}}\left[-A_{T}^{\mathbb{Q}}\right]-\mathscr{E}_{I}^{\mathbb{Q}}\right\}=-\inf _{\mathbb{Q} \in \mathscr{Q}_{\mathscr{S}, I}}\left\{\mathbb{E}^{\mathbb{Q}}\left[A_{T}^{\mathbb{Q}}\right]+\mathscr{E}_{I}^{\mathbb{Q}}\right\}<0
$$

which already induces model-independent arbitrage.

Let us recall the set-up in Section 3.3 . every option $\psi_{i}$ can actually be liquidly traded at time 0 ; that is, for each $i \in I$, we have $c_{i}^{\prime}(\eta)$ being a constant $p_{i} \in \mathbb{R}$. Hence,

$$
\mathscr{P}_{\mathscr{S}, I}=\mathscr{P}_{\mathscr{S},\left(p_{i}\right)_{i \in I}}:=\left\{\mathbb{Q} \in \mathscr{P}_{\mathscr{S}}: \mathbb{E}^{\mathbb{Q}}\left[\psi_{i}\right]=p_{i}, \forall i \in I\right\}
$$

Corollary 4.9 Suppose $\psi_{i}$ is continuous, $\left|\psi_{i}\right|$ satisfies (3.11), and $\psi_{i}$ can be liquidly traded at the price $p_{i} \in \mathbb{R}$, for all $i \in I$.

(i) No model-independent arbitrage under constraint $\mathscr{S}$ if and only if $\mathscr{P}_{\mathscr{S},\left(p_{i}\right)_{i \in I}} \neq \emptyset$.

(ii) Furthermore, suppose there is no portfolio constraint, i.e. $\mathscr{S}=\mathscr{H}$. Then, there is no model-independent arbitrage if and only if $\mathscr{M}_{\left(p_{i}\right)_{i \in I}} \neq \emptyset$.

Remark 4.10 Corollary 4.9 (ii) recovers [1. Theorem 1.3], for the case where tradable options at time 0 include at least vanilla calls of all maturities and strikes.

Remark 4.11 Among different model-independent versions of the fundamental theorem of asset pricing (FTAP), Theorem 4.8 and [1] Theorem 1.3] are unique in their ability to accommodate general collections of tradable options. While our framework deals with a wide range of tradable options beyond liquidly traded vanilla calls, [1] does not even assume that vanilla calls have to be tradable. On the other hand, while [1] implicitly assume that any tradable option is traded liquidly, we allow for less liquid options by taking into account their limit order books.

Also note that our method differs largely from that in [1]. Techniques in functional analysis, which involves the use of Hahn-Banach theorem, are used to establish [1] Theorem 1.3]; see [1] Proposition 2.3]. In our case, we first derive a superhedging duality in Theorem 3.14 via optimal transport. Leveraging on this duality, we obtain the desired FTAP in Theorem 4.8 
4.1 Comparison with the classical theory

In the classical theory, a physical measure $\mathbb{P}$ on $\left(\Omega, \mathscr{F}_{T}\right)$ is a priori given. We say there is no-arbitrage under $\mathbb{P}$ with the constraint $\mathscr{S}$ if, for any $\Delta \in \mathscr{S},(\Delta \cdot S)_{T} \geq 0$ $\mathbb{P}$-a.s. implies $(\Delta \cdot S)_{T}=0 \mathbb{P}$-a.s.

Consider the positive cone $\mathscr{K}:=\{\lambda \Delta \mid \Delta \in \mathscr{S}, \lambda \geq 0\}$ generated by $\mathscr{S}$. For all $t=1, \ldots, T$, we define $\mathscr{S}_{t}:=\left\{\Delta_{t} \mid \Delta \in \mathscr{S}\right\}, \mathscr{K}_{t}:=\left\{\Delta_{t} \mid \Delta \in \mathscr{K}\right\}$, and introduce

$$
\begin{aligned}
N_{t} & :=\left\{\eta \in L^{0}\left(\Omega, \mathscr{F}_{t-1}, \mathbb{P} ; \mathbb{R}^{d}\right): \eta \cdot\left(S_{t}-S_{t-1}\right)=0 \mathbb{P} \text {-a.s. }\right\}, \\
N_{t}^{\perp} & :=\left\{\xi \in L^{0}\left(\Omega, \mathscr{F}_{t-1}, \mathbb{P} ; \mathbb{R}^{d}\right): \xi \cdot \eta=0 \mathbb{P} \text {-a.s. for all } \eta \in N_{t}\right\} .
\end{aligned}
$$

By [21, Lemma 1.66], every $\xi \in L^{0}\left(\Omega, \mathscr{F}_{t-1}, \mathbb{P} ; \mathbb{R}^{d}\right)$ has a unique decomposition $\xi=\eta+\xi^{\perp}$, with $\eta \in N_{t}$ and $\xi^{\perp} \in N_{t}^{\perp}$. We denote by $\hat{\mathscr{S}}_{t}$ and $\hat{\mathscr{K}}_{t}$ the closures of $\mathscr{S}_{t}$ and $\mathscr{K}_{t}$, respectively, in $L^{0}\left(\Omega, \mathscr{F}_{t-1}, \mathbb{P} ; \mathbb{R}^{d}\right)$. The following characterization of no-arbitrage under $\mathbb{P}$ is taken from [21, Theorem 9.9].

Proposition 4.12 Suppose that for all $t=1, \ldots, T$,

$$
\mathscr{S}_{t}=\hat{\mathscr{S}}_{t}, \quad \hat{\mathscr{K}}_{t} \cap L^{\infty}\left(\Omega, \mathscr{F}_{t-1}, \mathbb{P} ; \mathbb{R}^{d}\right) \subseteq \mathscr{K}_{t}, \quad \text { and } \quad \xi^{\perp} \in \mathscr{S}_{t} \text { for any } \xi \in \mathscr{S}_{t}
$$

Then, there is no arbitrage under $\mathbb{P}$ with the constraint $\mathscr{S}$ if and only if

$$
\begin{array}{r}
\mathscr{P}_{\mathscr{S}}(\mathbb{P}):=\left\{\mathbb{Q} \approx \mathbb{P}: S_{t} \in L^{1}(\mathbb{Q}) \text { and }\left\{(\Delta \cdot S)_{t}\right\}_{t=0}^{T} \text { is a local } \mathbb{Q}\right. \text {-supermartingale, } \\
\forall \Delta \in \mathscr{S}\} \neq \emptyset .
\end{array}
$$

Theorem 4.8 can be viewed as a generalization of Proposition 4.12 to a modelindependent setting. There is, however, a notable discrepancy: the closedness assumption (4.1) is no longer needed in Theorem 4.8. In the following, we provide a detailed illustration of this discrepancy in a simple example.

A typical example showing that condition (4.1) is indispensable for Proposition 4.12 is a one-period model containing two risky assets $\left(S^{1}, S^{2}\right)$, with the collection of constrained strategies

$$
\mathscr{S}:=\left\{\left(\Delta^{1}, \Delta^{2}\right) \in \mathbb{R}^{2} \mid\left(\Delta^{1}\right)^{2}+\left(\Delta^{2}-1\right)^{2} \leq 1\right\} .
$$

One easily sees that 4.1 is not satisfied, as $\hat{\mathscr{K}}_{1} \cap L^{\infty}\left(\Omega, \mathscr{F}_{0}, \mathbb{P}\right)=\overline{\mathscr{K}}=\left\{\Delta^{2} \geq 0\right\}$ is not contained in $\mathscr{K}_{1}=\mathscr{K}=\{(0,0)\} \cup\left\{\Delta^{2}>0\right\}$. At time 0 , suppose $\left(S_{0}^{1}, S_{0}^{2}\right)=(1,1)$, and we obtain, by analyzing market data, that a reasonable pricing measure $\mathbb{Q}$ should be such that

$S_{1}^{1}$ is uniformly distributed on $[1,2]$, and $S_{1}^{2}$ is concentrated solely on $\{0\}$.

Under the classical framework, the physical measure $\mathbb{P}$ should satisfy (4.2), so that any pricing measure $\mathbb{Q} \approx \mathbb{P}$ admits the same property. Given $\Delta \in \mathscr{S}$, it can be checked that if $(\Delta \cdot S)_{T}=\Delta^{1}\left(S_{1}^{1}-1\right)-\Delta^{2} \geq 0 \mathbb{P}$-a.s., then $\Delta^{1}=\Delta^{2}=0$. There is therefore no arbitrage under $\mathbb{P}$ with the constraint $\mathscr{S}$. However, as observed in [4, Example 2.1], $\mathscr{P}_{\mathscr{S}}(\mathbb{P})$ is empty. Indeed, given $\mathbb{Q} \approx \mathbb{P}$, since $\mathbb{E}^{\mathbb{Q}}\left[S_{1}^{1}-1\right]>0$, by taking $\Delta \in \mathscr{S}$ with $\Delta^{2} / \Delta^{1}<\mathbb{E}^{\mathbb{Q}}\left[S_{1}^{1}-1\right]$, one gets $\mathbb{E}^{\mathbb{Q}}\left[(\Delta \cdot S)_{T}\right]=\Delta^{1} \mathbb{E}^{\mathbb{Q}}\left[S_{1}^{1}-1\right]-\Delta^{2}>0$. 
Under our model-independent framework, (4.2) is reflected through market prices of vanilla calls $\left(S_{1}^{1}-K\right)^{+}$and $\left(S_{1}^{2}-K\right)^{+}$, for all $K \geq 0$. That is, $\Pi$ in (2.2) is the collection $\{\mathbb{Q} \in \mathscr{P}(\Omega):(4.2)$ is satisfied $\}$. Given $\mathbb{Q} \in \Pi$, since $\mathbb{E}^{\mathbb{Q}}\left[S_{1}^{1}\right]=3 / 2$, by taking $\Delta \in \mathscr{S}$ with $\Delta^{2} / \Delta^{1}<1 / 2$, one gets $\mathbb{E}^{\mathbb{Q}}\left[(\Delta \cdot S)_{T}\right]=\Delta^{1} \mathbb{E}^{\mathbb{Q}}\left[S_{1}^{1}-1\right]-\Delta^{2}>0$. This shows that $\mathscr{P}_{\mathscr{S}}=\emptyset$. Note that this does not violate Theorem 4.8, as there is model-independent arbitrage. To see this, consider trading dynamically with $\Delta \in \mathscr{S}$ satisfying $\Delta^{2}<\varepsilon \Delta^{1}$ for some $\varepsilon \in(0,1)$, and holding a static position $u_{1}^{1}$ given by $-\Delta^{1}\left(S_{1}^{1}-\varepsilon\right)^{+}+(1+\varepsilon) \Delta^{1} \in \mathscr{C}$. Observe that the initial wealth required is

$$
\int_{\mathbb{R}_{+}} u_{1}^{1}(x) d \mu_{1}^{1}(x)=-\Delta^{1}(3 / 2-\varepsilon)+(1+\varepsilon) \Delta^{1}=(2 \varepsilon-1 / 2) \Delta^{1},
$$

while the terminal wealth is always strictly positive: for any $\left(S_{1}^{1}, S_{1}^{2}\right) \in \mathbb{R}_{+}^{2}$,

$$
u_{1}^{1}+(\Delta \cdot S)_{1}= \begin{cases}2 \varepsilon \Delta^{1}+\Delta^{2}\left(S_{1}^{2}-1\right)>\varepsilon \Delta^{1}-\Delta^{2}>0, & \text { if } S_{1}^{1} \geq \varepsilon \\ \left(\varepsilon \Delta^{1}-\Delta^{2}\right)+\left(\Delta^{1} S_{1}^{1}+\Delta^{2} S_{1}^{2}\right)>0, & \text { if } S_{1}^{2}<\varepsilon\end{cases}
$$

By taking $\varepsilon \in(0,1 / 4]$, we have the required initial wealth no greater than 0 , and thus obtain model-independent arbitrage.

Remark 4.13 In the model-independent setting, we may hold static positions in vanilla calls $\left(S_{1}^{i}-K\right)^{+}$for all $i \in\{1,2\}$ and $K>0$, in addition to trading $S=\left(S^{1}, S^{2}\right)$ dynamically. This additional flexibility, unavailable under the classical framework, allows us to construct the arbitrage in (4.3).

It is of interest to see if (4.1) can be relaxed in the classical case, when enough tradable options are available at time 0. Recently, with additional tradable options, [4] obtained a result similar to Proposition 4.12 with a collection $\mathscr{P}$ of possible physical measures a priori given. However, since their method allows for only finitely many tradable options, a closedness assumption similar to (4.1) is still assumed.

\subsection{Optimal arbitrage under a model-independent framework}

In view of Theorem 3.14 and Proposition 3.17, the problems of superhedging and risk-measuring are well-defined as long as $\mathscr{Q}_{\mathscr{S}, I} \neq \emptyset$, which is weaker than the noarbitrage condition $\mathscr{P}_{\mathscr{S}, I} \neq \emptyset$. It is therefore of interest to provide characterizations for the condition $\mathscr{Q}_{\mathscr{S}, I} \neq \emptyset$.

Definition 4.14 Consider

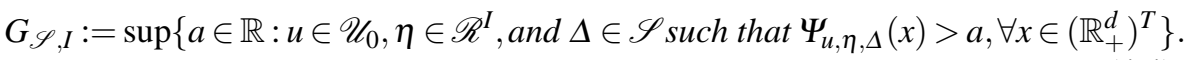

By definition, $G_{\mathscr{S}_{\text {,I }}} \geq 0$. If $G_{\mathscr{S}_{\text {, I }}}>0$, we say it is the (model-independent) optimal arbitrage profit.

The notion of optimal arbitrage goes back to [17], where the authors studied the highest return one can achieve relative to the market capitalization in a diffusion setting. Generalization to semimartingale models and model uncertainty settings have been 
done in [10] and [18], respectively. Our definition above is similar to the formulation in [10, Section 3]. It is straightforward from the definitions of $D(0)$ and $G_{\mathscr{S}, I}$ that

$$
G_{\mathscr{S}, I}=-D(0)=\inf _{\mathbb{Q} \in \mathscr{Q}_{\mathscr{S}, I}}\left\{\mathbb{E}^{\mathbb{Q}}\left[A_{T}^{\mathbb{Q}}\right]+\mathscr{E}_{I}^{\mathbb{Q}}\right\} .
$$

This immediately yields the following result.

Proposition 4.15 (i) $G_{\mathscr{S}, I}=0 \Longleftrightarrow \mathscr{P}_{\mathscr{S}, I} \neq \emptyset . \quad$ (ii) $G_{\mathscr{S}_{,}}<\infty \Longleftrightarrow \mathscr{Q}_{\mathscr{S}, I} \neq \emptyset$.

\section{Examples}

In this section, we will provide several concrete examples of the collection $\mathscr{S}$ of constrained trading strategies. An example which illustrates the effect of an additional tradable, yet less lquid, option will also be given. It will be convenient to keep in mind the relation $\mathscr{M} \subseteq \mathscr{P}_{\mathscr{S}} \subseteq \mathscr{Q}_{\mathscr{S}} \subseteq \Pi$, obtained from Remarks 4.3 and 4.5 Let us start with analyzing $\mathscr{Q}_{\mathscr{S}}$ further.

Proposition 5.1 Let $\mathscr{S}^{\infty}$ contain all nonnegative bounded trading strategies in $\mathscr{H}$.

(i) For any $\mathbb{Q} \in \mathscr{Q}_{\mathscr{S}},\left\{S_{t}\right\}_{t=0}^{T}$ is a $\mathbb{Q}$-supermartingale.

(ii) Furthermore, if trading strategies in $\mathscr{S}^{\infty}$ are uniformly bounded from below, i.e.

$$
\sup _{\Delta \in \mathscr{S}} \sup _{x \in\left(\mathbb{R}_{+}^{T}\right)^{d}}\left|\Delta^{-}(x)\right| \leq C \quad \text { for some } C>0,
$$

then $\mathscr{Q}_{\mathscr{S}}=\left\{\mathbb{Q} \in \Pi:\left\{S_{t}\right\}_{t=0}^{T}\right.$ is a $\mathbb{Q}$-supermartingale $\}$.

Proof. (i) Given $\mathbb{Q} \in \mathscr{Q}_{\mathscr{S}}$, if $\left\{S_{t}\right\}_{t=0}^{T}$ is not a $\mathbb{Q}$-supermartingale, there must exist $n^{*} \in\{1, \ldots, d\}$ and $t^{*} \in\{0, \ldots, T-1\}$ such that $\mathbb{Q}\left(\mathbb{E}^{\mathbb{Q}}\left[S_{t^{*}+1}^{n^{*}} \mid \mathscr{F}_{t^{*}}\right]-S_{t^{*}}^{n^{*}}>0\right)>0$. We then deduce from (3.3) that $\mathbb{Q}\left(A_{t^{*}+1}^{\mathbb{Q}}-A_{t^{*}}^{\mathbb{Q}}=\infty\right)>0$. This implies $\mathbb{E}^{\mathbb{Q}}\left[A_{T}^{\mathbb{Q}}\right]=\infty$, a contradiction to $\mathbb{Q} \in \mathscr{Q}_{\mathscr{S}}$.

(ii) Let $\mathbb{Q} \in \Pi$ be such that $\left\{S_{t}\right\}_{t=0}^{T}$ is a $\mathbb{Q}$-supermartingale. It can be easily checked that $\left\{(\Delta \cdot S)_{t}\right\}_{t=0}^{T}$ is a $\mathbb{Q}$-supermartingale, for any nonnegative bounded trading strategies $\Delta \in \mathscr{H}$. By [3.5),

$$
\begin{aligned}
\mathbb{E}^{\mathbb{Q}}\left[A_{T}^{\mathbb{Q}}\right] & =\sup _{\Delta \in \mathscr{S}^{\infty}}\left(\mathbb{E}^{\mathbb{Q}}\left[\left(\Delta^{+} \cdot S\right)_{T}\right]-\mathbb{E}^{\mathbb{Q}}\left[\left(\Delta^{-} \cdot S\right)_{T}\right]\right) \\
& \leq \sup _{\Delta \in \mathscr{S}^{\infty}} \mathbb{E}^{\mathbb{Q}}\left[\left|\left(\Delta^{-} \cdot S\right)_{T}\right|\right] \leq 2 C \sum_{t=1}^{T} \sum_{n=1}^{d}\left(\mathbb{E}^{\mathbb{Q}}\left[S_{t+1}^{n}\right]+\mathbb{E}^{\mathbb{Q}}\left[S_{t}^{n}\right]\right)<+\infty,
\end{aligned}
$$

which implies that $\mathbb{Q} \in \mathscr{Q}_{\mathscr{S}}$.

Example 5.2 (Shortselling constraint) Given $c_{t}^{n} \geq 0$ for each $t=0, \ldots, T-1$ and $n=1, \ldots, d$, we see from Remark 2.9 (with $K_{t}=\prod_{n}\left[-c_{t}^{n}, \infty\right)$ for all $t$ ) that

$$
\mathscr{S}:=\left\{\Delta \in \mathscr{H}: \Delta_{t}^{n} \geq-c_{t}^{n}, \forall t=0, \ldots, T-1 \text { and } n=1, \ldots, d\right\} .
$$


satisfies Definition 2.7 By Proposition 5.1] we have

$$
\mathscr{Q}_{\mathscr{S}}=\left\{\mathbb{Q} \in \Pi:\left\{S_{t}\right\}_{t=0}^{T} \text { is a } \mathbb{Q} \text {-supermartingale }\right\} .
$$

Furthermore, if $c_{t}^{n}>0$ for all t and $n$, then $\mathscr{M}=\mathscr{P}_{\mathscr{S}}$ by virtue of Remark 4.3 If there exists $n \in\{1, \ldots, d\}$ such that $c_{t}^{n}=0$ for all $t$, then $\left\{S_{t}^{n}\right\}_{t=0}^{T}$ is a $\mathbb{Q}$-supermartingale for all $\mathbb{Q} \in \mathscr{P}_{\mathscr{S}}$. Thus, if $c_{t}^{n}=0$ for all $t$ and $n$, then $\mathscr{P}_{\mathscr{S}}=\mathscr{Q}_{\mathscr{S}}$.

Example 5.3 (Relative-drawdown constraint) Let $x_{0}^{n}>0$ for $n=1, \ldots, d$. For each $x \in\left(\mathbb{R}_{+}^{d}\right)^{T}, t=1, \ldots, T$ and $n \in\{1, \ldots, d\}$, consider the running maximum $x_{t}^{*, n}$ given by $\max \left\{x_{0}^{n}, x_{1}^{n}, \ldots, x_{t}^{n}\right\}$. Then, define the relative drawdown process $\left\{\tilde{x}_{t}: t=0, \ldots, T\right\}$ by $\tilde{x}_{t}:=\left(x_{t}^{1} / x_{t}^{*, 1}, \ldots, x_{t}^{d} / x_{t}^{*, d}\right)$. For each $n=1, \ldots, d$, take two continuous functions $a^{n}:[0,1]^{d} \mapsto(-\infty, 0]$ and $b^{n}:[0,1]^{d} \mapsto[0, \infty)$, and introduce

$$
\begin{aligned}
\mathscr{S} & :=\left\{\Delta \in \mathscr{H}: a^{n}\left(\tilde{x}_{t}\right) \leq \Delta_{t}^{n}\left(x_{t}\right) \leq b^{n}\left(\tilde{x}_{t}\right), \forall t=0, \ldots, T-1, n=1, \ldots, d\right\} . \\
& =\left\{\Delta \in \mathscr{H}: \Delta_{t} \in K_{t}, \forall t=0, \ldots, T-1\right\}, \text { with } K_{t}:=\prod_{i=1}^{d}\left[a^{n}\left(\tilde{x}_{t}\right), b^{n}\left(\tilde{x}_{t}\right)\right] .
\end{aligned}
$$

Since $K_{t}$ satisfies (2.6), Remark 2.9] shows that $\mathscr{S}$ satisfies Definition 2.7 Thanks to Remark 3.5 we have $\mathscr{Q}_{\mathscr{S}}=\Pi$.

Example 5.4 (Non-tradable assets) Suppose certain risky assets are not tradable. In markets of electricity and foreign exchange rates, for example, people trade options written on the non-tradable underlying. More precisely, let $d^{\prime} \in\{1, \ldots, d\}$ and set

$$
\mathscr{S}:=\left\{\Delta \in \mathscr{H}: \Delta_{t}^{n} \equiv 0, \quad \text { for all } t=1, \ldots, T \text { and } n=1, \ldots, d^{\prime}\right\} .
$$

By a similar argument in Proposition 5.1. one can show that

$$
\mathscr{P}_{\mathscr{S}}=\mathscr{Q}_{\mathscr{S}}=\left\{\mathbb{Q} \in \Pi:\left\{S_{t}^{n}\right\}_{t=1}^{T} \text { is a } \mathbb{Q} \text {-martingale, for all } n=d^{\prime}+1, \ldots, d\right\} .
$$

By Theorem 4.8 there is no model-independent arbitrage if and only if there exists $\mathbb{Q} \in \Pi$ under which all tradable assets are martingales. We can also modify this example by imposing additional constraint on the tradable assets satisfying Definition 2.7 In this case, Theorem 4.8 suggests that there is no arbitrage if and only if there is no arbitrage in the market consisting of tradable assets only.

Example 5.5 (Less Liquid Option) Consider a two-period model with one risky asset starting from $S_{0}=2$. We assume as in [5] Section 4.2] that the marginal distributions for $S_{1}$ and $S_{2}$ are given by

$$
d \mu_{1}(x)=\frac{1}{2} 1_{[1,3]}(x) d x, \quad d \mu_{2}(x)=\frac{x}{3} 1_{[0,1]}(x) d x+\frac{1}{3} 1_{[1,3]}(x) d x+\frac{4-x}{3} 1_{[3,4]}(x) d x .
$$

In addition to vanilla calls, we assume that a forward-start straddle with payoff $\psi(S)=\left|S_{2}-S_{1}\right|$ is also tradable at time 0 , whose unit price for trading $\eta$ units is given by $p(\eta):=\infty 1_{\{\eta>1\}}+a 1_{\{0 \leq \eta \leq 1\}}+b 1_{\{-1 \leq \eta<0\}}$, where $0 \leq b \leq a$, and we take $0 \cdot \infty=0$. We assume that the portfolio constraint $\mathscr{S}$ satisfies $\mathscr{Q}_{\mathscr{S}}=\mathscr{M}$. This readily covers the no-constraint case, as explained in Remark 3.12 Moreover, it also 
includes the shortselling constraint as in Example 5.2 To see this, note from Example 5.2 that $\mathscr{Q}_{\mathscr{S}}=\left\{\mathbb{Q} \in \Pi:\left\{S_{t}\right\}_{t=0}^{T}\right.$ is a $\mathbb{Q}$-supermartingale $\}$. But since $\mathbb{Q} \in \Pi$ implies that $\mathbb{E}^{\mathbb{Q}}\left[S_{1}\right]=\mathbb{E}^{\mathbb{Q}}\left[S_{2}\right]=2$ (computed from $\mu_{1}$ and $\mu_{2}$ ), every $\mathbb{Q} \in \mathscr{Q}_{\mathscr{S}}$ is actually a martingale. We thus obtain $\mathscr{Q}_{\mathscr{S}}=\mathscr{M}$.

We intend to price an exotic option with payoff $\Phi\left(x_{1}, x_{2}\right)=\left(x_{2}-x_{1}\right)^{2}$. Our goal is to see how using the additional option $\psi$ in static hedging affects the superhedging price of $\Phi$. First, for any $\mathbb{Q} \in \mathscr{M}$, the martingale property of $S$ implies $\mathbb{E}^{\mathbb{Q}}\left[\left(S_{2}-S_{1}\right)^{2}\right]=\mathbb{E}^{\mathbb{Q}}\left[S_{2}^{2}\right]-\mathbb{E}^{\mathbb{Q}}\left[S_{1}^{2}\right]=\frac{1}{2}$, which is obtained solely from $\mu_{1}$ and $\mu_{2}$. Since $\mathscr{Q}_{\mathscr{S}}=\mathscr{M}$, Proposition 3.10 gives $D_{\emptyset}(\Phi)=\frac{1}{2}$. On the other hand, $\mathscr{Q}_{\mathscr{S}}=\mathscr{M}$ and $\mathscr{E}_{\{\psi\}}^{\mathbb{Q}}<\infty$ for all $\mathbb{Q} \in \mathscr{M}$ (see Remark 3.8$)$ imply that $\mathscr{Q}_{\mathscr{S}, I}=\mathscr{M}$. Theorem 3.14 thus yields

$$
\begin{aligned}
D(\Phi) & =\sup _{\mathbb{Q} \in \mathscr{M}}\left(\mathbb{E}^{\mathbb{Q}}\left[\left(S_{2}-S_{1}\right)^{2}\right]-\mathscr{E}_{I}^{\mathbb{Q}}\right)=\frac{1}{2}-\inf _{\mathbb{Q} \in \mathscr{M}} \sup _{\eta \in[-1,1]} \eta\left(\mathbb{E}^{\mathbb{Q}}\left[\left|S_{2}-S_{1}\right|\right]-p(\eta)\right) \\
& =\frac{1}{2}-\sup _{\eta \in[-1,1]} \eta\left(\inf _{\mathbb{Q} \in \mathscr{M}} \mathbb{E}^{\mathbb{Q}}\left[\left|S_{2}-S_{1}\right|\right]-p(\eta)\right),
\end{aligned}
$$

where in the second line we used Lemma 3.9. Recalling from [5. Section 4.2] that $\inf _{\mathbb{Q} \in \mathscr{M}} \mathbb{E}^{\mathbb{Q}}\left[\left|S_{2}-S_{1}\right|\right]=\frac{1}{3}$, we get $D(\Phi)=\frac{1}{2}-\max \left\{\left(\frac{1}{3}-a\right)^{+},\left(b-\frac{1}{3}\right)^{+}\right\}$.

\section{Bounded constraints without adapted convexity}

In this section, we extend the main results of this paper, Theorems 3.14 and 4.8 , to a class of constraints which does not satisfy adapted convexity (Definition 2.7 (ii)), but instead admits additional boundedness property. Motivations behind this include Gamma constraint, which will be discussed in Sections 6.1

Definition 6.1 $\mathscr{S}$ is a collection of trading strategies satisfying Definition 2.7(i) and (iii), while condition (ii) is replaced by the following:

(ii) ${ }^{\prime}$ (Boundedness) For any $\Delta \in \mathscr{S}, \exists c>0$ such that $\left|\Delta_{t}(x)\right| \leq c$ for all $x \in\left(\mathbb{R}_{+}^{d}\right)^{t}$ and $t \in\{0, \ldots, T-1\}$ (i.e. $\mathscr{S}=\mathscr{S}^{\infty}$ ).

Under current setting, Lemma 3.2 does not hold anymore, and thus the upper variation process $A_{t}^{\mathbb{Q}}$ is no longer useful. We adjust the definitions of $\mathscr{Q}_{\mathscr{S}}$ and $\mathscr{P}_{\mathscr{S}}$ accordingly.

Definition 6.2 For any $\mathbb{Q} \in \Pi$, we define

$$
C^{\mathbb{Q}}:=\sup _{\Delta \in \mathscr{S}} \mathbb{E}^{\mathbb{Q}}\left[(\Delta \cdot S)_{T}\right]
$$

In analogy to $\mathscr{Q}_{\mathscr{S}}$ in Definition 3.4 and the characterization of $\mathscr{P}_{\mathscr{S}}$ in Lemma 4.4 we define

$$
\mathscr{Q}_{\mathscr{S}}^{\prime}:=\left\{\mathbb{Q} \in \Pi: C^{\mathbb{Q}}<+\infty\right\} \quad \text { and } \quad \mathscr{P}_{\mathscr{S}}^{\prime}:=\left\{\mathbb{Q} \in \Pi: C^{\mathbb{Q}}=0\right\} .
$$


Recall from (2.4) that $\mathscr{S}_{c}$ denotes the collection of $\Delta \in \mathscr{S}$ with $\Delta_{t}:\left(\mathbb{R}_{+}^{d}\right)^{t} \mapsto \mathbb{R}^{d}$ continuous for all $t=1, \ldots, T$. Using the arguments in Lemma3.3 gives

$$
C^{\mathbb{Q}}=\sup _{\Delta \in \mathscr{S}_{c}} \mathbb{E}^{\mathbb{Q}}\left[(\Delta \cdot S)_{T}\right], \quad \forall \mathbb{Q} \in \Pi .
$$

By (6.1), 6.3), and similar arguments in Proposition 3.10 (with $\mathbb{E}^{\mathbb{Q}}\left[A_{T}^{\mathbb{Q}}\right]$ replaced by $\left.C^{\mathbb{Q}}\right)$, we obtain:

Proposition 6.3 Suppose $\mathscr{S}$ satisfies Definition 6.1 Let $\Phi:\left(\mathbb{R}_{+}^{d}\right)^{T} \mapsto \mathbb{R}$ be a measurable function for which there exists $K>0$ such that (3.11) holds.

(i) We have

$$
P_{\emptyset}^{\prime}(\Phi):=\sup _{\mathbb{Q} \in \mathscr{Q}_{\mathscr{S}}^{\prime}} \mathbb{E}^{\mathbb{Q}}[\Phi]-C^{\mathbb{Q}} \leq D_{\emptyset}(\Phi)
$$

(ii) Furthermore, if $\Phi$ is upper semicontinuous, then $P_{\emptyset}^{\prime}(\Phi)=D_{\emptyset}(\Phi)$.

(iii) If $\Phi$ is upper semicontinuous and $\mathscr{Q}_{\mathscr{S}}^{\prime} \neq \emptyset$, there exists $\mathbb{Q}^{*} \in \mathscr{Q}_{\mathscr{S}}^{\prime}$ such that $P_{\emptyset}^{\prime}(\Phi)=\mathbb{E}^{\mathbb{Q}^{*}}[\Phi]-C^{\mathbb{Q}^{*}}$.

Remark 6.4 Under adapted convexity (Definition 2.7 (ii)), Lemma 3.2 asserts that $\mathbb{E}^{\mathbb{Q}}\left[A_{T}^{\mathbb{Q}}\right]=C^{\mathbb{Q}}$. This need not be true in general. For any $\mathbb{Q} \in \Pi$, we observe from Lemma 3.2 that in general $\mathbb{E}^{\mathbb{Q}}\left[A_{T}^{\mathbb{Q}}\right] \geq \sup _{\Delta \in \mathscr{S}^{\infty}} \mathbb{E}^{\mathbb{Q}}\left[(\Delta . S)_{T}\right]=C^{\mathbb{Q}}$. This in particular implies that $P_{\emptyset}^{\prime}(\Phi) \geq P_{\emptyset}(\Phi)$.

We now include the collection of options $\left\{\psi_{i}\right\}_{i \in I}$ in the superhedging strategy. Recalling the definition of $\mathscr{E}_{I}^{\mathbb{Q}}$ from $(3.6)$, we consider the collection of measures $\mathscr{Q}_{\mathscr{S}, I}^{\prime}:=\left\{\mathbb{Q} \in \mathscr{Q}_{\mathscr{S}}^{\prime}: \mathscr{E}_{I}^{\mathbb{Q}}<\infty\right\}$, and define

$$
P^{\prime}(\Phi):=\sup _{\mathbb{Q} \in \mathscr{Q}_{\mathscr{S}}^{\prime}} \mathbb{E}^{\mathbb{Q}}[\Phi]-C^{\mathbb{Q}}-\mathscr{E}_{I}^{\mathbb{Q}}
$$

The following result follows from a straightforward adjustment of Theorem 3.14

Proposition 6.5 Suppose $\mathscr{S}$ satisfies Definition 6.1] $\psi_{i}$ is continuous and $\left|\psi_{i}\right|$ satisfies (3.11) for all $i \in I$. Then, for any upper semicontinuous function $\Phi:\left(\mathbb{R}_{+}^{d}\right)^{T} \mapsto \mathbb{R}$ satisfying (3.11), we have $D(\Phi)=P^{\prime}(\Phi)$, with $D$ and $P^{\prime}$ defined as in (3.2) and (6.4). Moreover, if $\mathscr{Q}_{\mathscr{S}, I}^{\prime} \neq \emptyset$, the supremum in (6.4) is attained at some $\mathbb{Q}^{*} \in \mathscr{Q}_{\mathscr{S}, I}^{\prime}$

To derive the FTAP, we consider the collection of measures

$$
\mathscr{P}_{\mathscr{S}, I}^{\prime}:=\left\{\mathbb{Q} \in \mathscr{P}_{\mathscr{S}}^{\prime}: c_{i}^{\prime}(0-) \leq \mathbb{E}^{\mathbb{Q}}\left[\psi_{i}\right] \leq c_{i}^{\prime}(0+) \text {, for all } i \in I\right\} \text {. }
$$

By (6.3), the same arguments in Lemma 4.7 (with $\mathbb{E}^{\mathbb{Q}}\left[A_{T}^{\mathbb{Q}}\right]$ replaced by $C^{\mathbb{Q}}$ ) yields:

$$
\mathscr{P}_{\mathscr{S}, I}^{\prime}=\emptyset \Longrightarrow \inf _{\mathbb{Q} \in \mathscr{Q}_{\mathscr{S}}^{\prime}}\left\{C^{\mathbb{Q}}+\mathscr{E}_{I}^{\mathbb{Q}}\right\}>0
$$

On strength of (6.5) and Proposition 6.5, we may argue as in Theorem 4.8 and Proposition 4.15 (with $\mathbb{E}^{\mathbb{Q}}\left[A_{T}^{\mathbb{Q}}\right]$ replaced by $C^{\mathbb{Q}}$ ) to establish the following.

Proposition 6.6 Suppose $\mathscr{S}$ satisfies Definition 6.1 Then,

(i) No model-independent arbitrage under the constraint $\mathscr{S}$ if and only if $\mathscr{P}_{\mathscr{S}, I}^{\prime} \neq \emptyset$.

(ii) Optimal arbitrage profit is finite under the constraint $\mathscr{S}$ (i.e. $G_{\mathscr{S}, I}<\infty$ ) if and only if $\mathscr{Q}_{\mathscr{S}, I}^{\prime} \neq \emptyset$. 
6.1 Gamma constraint

Given $\Gamma=\left(\Gamma_{1}, \ldots, \Gamma_{d}\right) \in \mathbb{R}_{+}^{d}$, we consider the collection of trading strategies

$$
\mathscr{S}_{\Gamma}:=\left\{\Delta \in \mathscr{H}:\left|\Delta_{t}^{n}-\Delta_{t-1}^{n}\right| \leq \Gamma_{n}, \forall t=0, \ldots, T-1, n=1, \ldots, d\right\},
$$

where we set $\Delta_{-1} \equiv 0 \in \mathbb{R}^{d}$. Observe that $\mathscr{S}_{\Gamma}$ does not admit adapted convexity (Definition 2.7 (ii)). Indeed, consider $\Delta \equiv 0$ and $\Delta^{\prime}:=\left\{1_{\{t=0\}} \Gamma+1_{\{t>0\}} 2 \Gamma\right\}_{t=0}^{T-1}$, both of which trivially lie in $\mathscr{S}_{\Gamma}$. Given a fixed $s \in\{1, \ldots, T-1\}$, the trading strategy $\tilde{\Delta}:=\left\{\Delta_{t} 1_{\{t<s\}}+\Delta_{t}^{\prime} 1_{\{t \geq s\}}\right\}_{t=0}^{T-1}$ does not belong to $\mathscr{S}_{\Gamma}$, as $\tilde{\Delta}_{s}-\tilde{\Delta}_{s-1}=2 \Gamma$. The constrained collection $\mathscr{S}_{\Gamma}$, instead, satisfies Definition 6.1.

Lemma 6.7 $\mathscr{S}_{\Gamma}$ satisfies Definition 6.1

Proof. It is trivial that $0 \in \mathscr{S}_{\Gamma}$. For each $\Delta \in \mathscr{S}_{\Gamma}$, since $\Delta_{t}=\sum_{j=0}^{t}\left(\Delta_{j}-\Delta_{j-1}\right)$, we have $\left|\Delta_{t}\right| \leq(t+1)|\Gamma|$, which shows that Definition 6.1 (ii) ${ }^{\prime}$ is satisfied. It remains to prove Definition 2.7(iii).

In view of Remark 2.9 , it follows from Lusin's theorem that for any $\mathbb{Q} \in \Pi$ and $\varepsilon>0$, there exist a closed set $D_{\varepsilon} \subseteq \Omega$ and a sequence of continuous functions $\Delta^{\varepsilon}(x)=\left\{\Delta_{t}^{\varepsilon}\left(x_{1}, \ldots, x_{t}\right)\right\}_{t=0}^{T-1}$ such that $\mathbb{Q}\left(D_{\varepsilon}\right)>1-\varepsilon$ and $\Delta=\Delta^{\varepsilon}$ on $D_{\varepsilon}$. That is, for all $t=1, \ldots, T-1, \Delta_{t}$ is a continuous function when it is restricted to the domain $\operatorname{proj}_{\left(\mathbb{R}_{+}^{d}\right)^{t}} D_{\varepsilon}:=\left\{x \in\left(\mathbb{R}_{+}^{d}\right)^{t}: \exists y \in\left(\mathbb{R}_{+}^{d}\right)^{T-t}\right.$ such that $\left.(x, y) \in D_{\varepsilon}\right\}$. In the following, by induction over time $t$, we will construct a continuous strategy $\bar{\Delta}^{\varepsilon} \in \mathscr{S}_{\Gamma, c}$. At time $t=0, \bar{\Delta}_{0}^{\varepsilon}:=\Delta_{0}$ is a constant in $\prod_{n=1}^{d}\left[-\Gamma_{n}, \Gamma_{n}\right]$, and therefore continuous. Fix $t \geq 1$. We assume that we have constructed continuous functions $\left\{\bar{\Delta}_{s}^{\varepsilon}:\left(\mathbb{R}_{+}^{d}\right)^{s} \mapsto \mathbb{R}^{d}\right\}_{s=0}^{t-1}$ such that $\bar{\Delta}_{s}^{\varepsilon}=\Delta_{s}$ on $\operatorname{proj}_{\left(\mathbb{R}_{+}^{d}\right)^{s}} D_{\varepsilon}$ and $\left|\bar{\Delta}_{s}^{\varepsilon}-\bar{\Delta}_{s-1}^{\varepsilon}\right| \leq \Gamma$ on $\left(\mathbb{R}_{+}^{d}\right)^{s} \backslash \operatorname{proj}_{\left(\mathbb{R}_{+}^{d}\right)^{s}} D_{\varepsilon}$, for any $s<t$. By the continuity of $\bar{\Delta}_{t-1}^{\varepsilon}$, the set-valued function defined by

$$
K_{t}\left(x_{1}, \ldots, x_{t}\right):=\left\{\begin{array}{lll}
\left\{\Delta_{t}\left(x_{1}, \ldots, x_{t}\right)\right\} & \text { on } & \operatorname{proj}_{\left(\mathbb{R}_{+}^{d}\right)^{t}} D_{\varepsilon} \\
\Delta_{t-1}\left(x_{1}, \ldots, x_{t-1}\right)+\prod_{n=1}^{d}\left[-\Gamma_{n}, \Gamma_{n}\right] & \text { on } & \left(\mathbb{R}_{+}^{d}\right)^{t} \backslash \operatorname{proj}_{\left(\mathbb{R}_{+}^{d}\right)^{t}} D_{\varepsilon}
\end{array}\right.
$$

satisfies (2.6) and thus admits a continuous selection ([31, Theorem 3.2" $]$ ); i.e. there is a continuous function $\bar{\Delta}_{t}^{\varepsilon}:\left(\mathbb{R}_{+}^{d}\right)^{t} \mapsto \mathbb{R}^{d}$ such that, $\bar{\Delta}_{t}^{\varepsilon}\left(x_{1}, \ldots, x_{t}\right) \in K_{t}\left(x_{1}, \ldots, x_{t}\right)$ for all $x \in \mathbb{R}_{+}^{t}$. Thus, we can construct $\bar{\Delta}^{\varepsilon} \in \mathscr{S}_{\Gamma, c}$, as required by Definition 2.7 (iii).

Proposition 6.8 $\mathscr{Q}_{\mathscr{S}_{\Gamma}}^{\prime}=\Pi$ and $\mathscr{P}_{\mathscr{S}_{\Gamma}}^{\prime}=\mathscr{M}$.

Proof. From the proof of Lemma6.7, every $\Delta \in \mathscr{S}_{\Gamma}$ is bounded by $c:=(T+1)|\Gamma|$. This gives $\mathscr{Q}_{\mathscr{S}_{\Gamma}}^{\prime}=\Pi$, by Remarks 3.5 For any $t \in\{0, \ldots, T-1\}$ and $A \in \mathscr{F}_{t}$, observe that $\Delta^{(+)}=\left\{\Delta_{s}^{+}\right\}_{s=0}^{T-1}:=+\Gamma 1_{A} 1_{\{s=t\}}$ and $\Delta^{(-)}=\left\{\Delta_{s}^{-}\right\}_{s=0}^{T-1}:=-\Gamma 1_{A} 1_{\{s=t\}}$ both belong to $\mathscr{S}_{\Gamma}$. Given $\mathbb{Q} \in \mathscr{P}_{\mathscr{S}_{\Gamma}}^{\prime}$, the definition of $\mathscr{P}_{\mathscr{S}_{\Gamma}}^{\prime}$ in 6.2) implies that $\mathbb{E}^{\mathbb{Q}}\left[\Gamma 1_{A}\left(S_{t+1}-S_{t}\right)\right]=0$. This readily implies $\mathbb{E}^{\mathbb{Q}}\left[S_{t+1} \mid \mathscr{F}_{t}\right]=S_{t}$, and thus $\mathbb{Q} \in \mathscr{M}$.

By Proposition 6.8 the following is a direct consequence of Proposition 6.6

Corollary 6.9 $\mathscr{S}_{\Gamma}$ satisfies the following: 
(i) There is no model-independent arbitrage under $\mathscr{S}_{\Gamma}$ if and only if $\mathscr{M}_{I} \neq \emptyset$.

(ii) Optimal arbitrage profit is finite under $\mathscr{S}_{\Gamma}$ (i.e. $\left.G_{\mathscr{S}_{\Gamma}, I}<\infty\right)$ if and only if $\mathscr{E}_{I}^{\mathbb{Q}}<\infty$ for some $\mathbb{Q} \in \Pi$.

Remark 6.10 By Theorem 4.8 Remark 3.12 and Corollary 6.9 we have equivalence between:

(i) There is model-independent arbitrage with $\Delta \in \mathscr{H}$ (i.e. the no-constraint case).

(ii) There is model-independent arbitrage with $\Delta \in \mathscr{S}_{\Gamma}$.

While these two arbitrage opportunities coexist, they are very different in terms of optimal arbitrage profit defined in (4.4). By Proposition 4.15. we see that $G_{\mathscr{H}}<\infty$ if and only if $\mathscr{Q}_{\mathscr{H}, I}=\left\{\mathbb{Q} \in \mathscr{M}: \mathscr{E}_{I}^{\mathbb{Q}}<\infty\right\} \neq \emptyset$, while $G_{\mathscr{S}_{\Gamma}}<\infty$ if and only if $\mathscr{Q}_{\mathscr{S}_{\Gamma}, I}^{\prime}=\left\{\mathbb{Q} \in \Pi: \mathscr{E}_{I}^{\mathbb{Q}}<\infty\right\} \neq \emptyset$.

\section{A An example related to definition 2.7(iii)}

In this appendix, we provide an example showing that if Definition 2.7 (iii) is not satisfied, the duality in Proposition 3.10 may fail. Let $d=1, T=2$ and $x_{0}=1$. Assume $\mu_{1}(d x)=\frac{1}{2} \delta_{1}(d x)+\frac{1}{2} \delta_{2}(d x)$ and $\mu_{2}(d x)=\delta_{2}(d x)$, where $\delta_{x}$ is the Dirac measure at $x \in \mathbb{R}$. Thus, $\Pi=\{\mathbb{Q}\}$ with $\mathbb{Q}\left(S_{1}=1, S_{2}=2\right)=\mathbb{Q}\left(S_{1}=2, S_{2}=2\right)=\frac{1}{2}$. Consider the collection of trading strategies

$$
\mathscr{S}=\left\{\Delta=\left(\Delta_{0}, \Delta_{1}\right): \Delta_{0} \equiv 0, \Delta_{1}(x)=\alpha 1_{\{x=1\}}(x) \text { for some } \alpha \in[0,1]\right\} .
$$

While $\mathscr{S}$ trivially satisfies Definition 2.7(i) and (ii), Definition 2.7(iii) does not hold. To see this, note that $\mathscr{S}_{c}^{\infty}=\{(0,0)\}$, and thus for any $\Delta \in \mathscr{S}$ with $\alpha>0$, we have $\mathbb{Q}(\Delta \neq(0,0))=1 / 2$. In order to superhedge the claim $\Phi\left(x_{1}, x_{2}\right) \equiv 0$, we need to find $n, m \in \mathbb{N}, a, b_{i}, c_{j} \in \mathbb{R}, K_{i}^{1}, K_{j}^{2} \geq 0$ and $\Delta \in \mathscr{S}$ such that for all $\left(x_{1}, x_{2}\right) \in \mathbb{R}_{+}^{2}$

$$
0 \leq a+\sum_{i=1}^{n} b_{i}\left(x_{1}-K_{i}^{1}\right)^{+}+\sum_{j=1}^{m} c_{j}\left(x_{2}-K_{j}^{2}\right)^{+}+\Delta_{0}\left(x_{1}-x_{0}\right)+\Delta_{1}\left(x_{1}\right)\left(x_{2}-x_{1}\right) .
$$

Since $\Delta_{0} \equiv 0$ and $\Delta\left(x_{1}\right)=\alpha 1_{\left\{x_{1}=1\right\}}$, the above inequality reduces to

$$
f_{\alpha}\left(x_{1}, x_{2}\right):=-\alpha 1_{\left\{x_{1}=1\right\}}\left(x_{1}\right)\left(x_{2}-x_{1}\right) \leq a+\sum_{i=1}^{n} b_{i}\left(x_{1}-K_{i}^{1}\right)^{+}+\sum_{j=1}^{m} c_{j}\left(x_{2}-K_{j}^{2}\right)^{+},
$$

for all $\left(x_{1}, x_{2}\right) \in \mathbb{R}_{+}^{2}$. Let $f_{\alpha}^{*}$ denote the upper semicontinuous envelope of $f_{\alpha}$. We observe that A.1 holds for $f_{\alpha}$ if and only if it holds also for $f_{\alpha}^{*}$. It follows that

$$
\begin{aligned}
D_{\emptyset}(0) & =\inf _{0 \leq \alpha \leq 1} D_{\emptyset}\left(f_{\alpha}\right)=\inf _{0 \leq \alpha \leq 1} D_{\emptyset}\left(f_{\alpha}^{*}\right)=\inf _{0 \leq \alpha \leq 1} P_{\emptyset}\left(f_{\alpha}^{*}\right) \\
& =\inf _{0 \leq \alpha \leq 1} \alpha \mathbb{E}^{\mathbb{Q}}\left[1_{\left\{S_{1}=1\right\}}\left(S_{1}\right)\left(S_{2}-S_{1}\right)^{-}\right]=0,
\end{aligned}
$$

where the third equality follows from Proposition 3.10 and the fourth equality is due to $f_{\alpha}^{*}=\alpha 1_{\left\{x_{1}=1\right\}}\left(x_{1}\right)\left(x_{2}-x_{1}\right)^{-}$. On the other hand, since

$$
A_{2}^{\mathbb{Q}}=\sup _{\alpha \in[0,1]} \alpha \mathbb{Q}\left(S_{1}=1\right)=\frac{1}{2}
$$


we have $P_{\emptyset}(0)=-\mathbb{E}^{\mathbb{Q}}\left[A_{2}^{\mathbb{Q}}\right]=-\frac{1}{2}$, which indicates a duality gap.

\section{References}

1. B. ACCIAio, M. Beiglböck, F. PEnkner, AND W. SCHAChermayer, A model-free version of the fundamental theorem of asset pricing and the super-replication theorem, Mathematical Finance, (2013).

2. J.-P. Aubin And H. Frankowska, Set-valued analysis, Modern Birkhäuser Classics, Birkhäuser Boston, Inc., Boston, MA, 2009. Reprint of the 1990 edition [MR1048347].

3. E. BAYRAKTAR, Y. ZHANG, AND Z. ZHOU, A note on the fundamental theorem of asset pricing under model uncertainty, Risks, 2 (2014), pp. 425-433.

4. E. BAYRAKTAR AND Z. ZHOU, On arbitrage and duality under model uncertainty and portfolio constraints, tech. rep., University of Michigan, 2014. Available at http://arxiv.org/abs/1402.2596.

5. M. Beiglböck, P. Henry-LABORdÈRE, AND F. PENKNER, Model-independent bounds for option prices-a mass transport approach, Finance Stoch., 17 (2013), pp. 477-501.

6. D. BERTSIMAS AND I. POPESCU, On the relation between option and stock prices: a convex optimization approach, Oper. Res., 50 (2002), pp. 358-374.

7. B. BOUCHARD AND M. NutZ, Arbitrage and duality in nondominated discrete-time models, Ann. Appl. Probab., 25 (2015), pp. 823-859.

8. H. Brown, D. Hobson, AND L. C. G. Rogers, Robust hedging of barrier options, Math. Finance, 11 (2001), pp. 285-314.

9. L. CARAssus, H. Pham, AND N. TOUZI, No arbitrage in discrete time under portfolio constraints, Math. Finance, 11 (2001), pp. 315-329.

10. H. N. CHAU AND P. TANKOV, Market models with optimal arbitrage, tech. rep., University of Padova and Université Paris-Diderot, 2013. Available at http://arxiv.org/abs/1312.4979.

11. X. Chen, G. Deelstra, J. Dhaene, And M. Vanmaele, Static super-replicating strategies for a class of exotic options, Insurance Math. Econom., 42 (2008), pp. 1067-1085.

12. A. M. G. COX AND J. OBŁóJ, Robust pricing and hedging of double no-touch options, Finance Stoch., 15 (2011), pp. 573-605.

13. J. CVITANiĆ AND I. KARATZAS, Hedging contingent claims with constrained portfolios, Ann. Appl. Probab., 3 (1993), pp. 652-681.

14. Y. DOLINSKY AND H. M. SONER, Martingale optimal transport and robust hedging in continuous time, Probability Theory and Related Fields, 160 (2014), pp. 391-427.

15. - Robust hedging with proportional transaction costs, Finance and Stochastics, 18 (2014), pp. 327-347.

16. B. DUPIRE, Pricing with a smile, Risk, 7 (1994), pp. 18-20.

17. D. Fernholz And I. Karatzas, On optimal arbitrage, Annals of Applied Probability, 20 (2010), pp. 1179-1204.

18. - Optimal arbitrage under model uncertainty, the Annals of Applied Probability, 21 (2011), pp. 2191-2225.

19. H. FÖLlMER AND D. KRAMKOV, Optional decompositions under constraints, Probab. Theory Related Fields, 109 (1997), pp. 1-25.

20. H. Föllmer AND A. SCHIED, Convex measures of risk and trading constraints, Finance Stoch., 6 (2002), pp. 429-447.

21. _ Stochastic finance, Walter de Gruyter \& Co., Berlin, extended ed., 2011. An introduction in discrete time.

22. A. GÖPFERT, H. RiAhi, C. TAMMER, AND C. ZĂLINESCU, Variational methods in partially ordered spaces, CMS Books in Mathematics/Ouvrages de Mathématiques de la SMC, 17, Springer-Verlag, New York, 2003.

23. P. HENRY-LABORDÈRE, Automated option pricing: Numerical methods, International Journal of Theoretical and Applied Finance, 16 (2013).

24. F. HIRSCH AND B. RoYNETTE, A new proof of Kellerer's theorem, ESAIM: Probability and Statistics, to appear, (2012).

25. D. HobSON, Robust hedging of the lookback option, Finance \& Stochastics, 2 (1998), pp. 329C-347.

26. D. HobSON, P. LAURENCE, AND T.-H. WANG, Static-arbitrage optimal subreplicating strategies for basket options, Insurance Math. Econom., 37 (2005), pp. 553-572. 
27. - Static-arbitrage upper bounds for the prices of basket options, Quant. Finance, 5 (2005), pp. 329-342.

28. E. Jouini And H. KAllal, Arbitrage in securities markets with short-sales constraints, Math. Finance, 5 (1995), pp. 197-232.

29. H. G. Kellerer, Duality theorems for marginal problems, Z. Wahrsch. Verw. Gebiete, 67 (1984), pp. 399-432.

30. P. LAURENCE AND T.-H. WANG, Sharp upper and lower bounds for basket options, Applied Mathematical Finance, 12 (2005), pp. 253-282.

31. E. Michael, Continuous selections. I, Annals of Mathematics. Second Series, 63 (1956), pp. 361382.

32. C. NAPP, The Dalang-Morton-Willinger theorem under cone constraints, J. Math. Econom., 39 (2003), pp. 111-126. Special issue on equilibrium with asymmetric information.

33. D. B. ROKHLIN, An extended version of the Dalang-Morton-Willinger theorem with convex portfolio constraints, Teor. Veroyatn. Primen., 49 (2004), pp. 503-521.

34. F. TERKElsen, Some minimax theorems, Math. Scand., 31 (1972), pp. 405-413 (1973).

35. C. Villani, Optimal transport, old and new, vol. 338 of Grundlehren der Mathematischen Wissenschaften [Fundamental Principles of Mathematical Sciences], Springer-Verlag, Berlin, 2009. 